\title{
Desenvolvimento de questionário de frequência alimentar a partir da avaliação do consumo alimentar habitual de pacientes com Doença Hepática Gordurosa não
}

\author{
Alcoólica \\ Development of a food frequency questionnaire based on the evaluation of the habitual dietary \\ intake of patients with Non-alcoholic Fatty Liver Disease \\ Desarrollo de cuestionario de frecuencia alimentaria a partir de la evaluación del consumo \\ alimentario habitual de pacientes con Enfermedad Hepática Grasa no Alcohólica
}

Recebido: 18/11/2021 | Revisado: 26/11/2021 | Aceito: 02/12/2021 | Publicado: 13/12/2021

\author{
Laís Spíndola Garcêz \\ ORCID: https://orcid.org/0000-0001-5818-9502 \\ Universidade do Estado da Bahia, Brasil \\ E-mail: 1sgarcez@uneb.br \\ Carla de Magalhães Cunha \\ ORCID: https://orcid.org/0000-0002-9644-7194 \\ Universidade Federal da Bahia, Brasil \\ E-mail: cdcunha@ufba.br \\ André Castro Lyra \\ ORCID: https://orcid.org/0000-0001-9010-8645 \\ Universidade Federal da Bahia, Brasil \\ E-mail: aclyra@live.com \\ Rosangela Passos de Jesus \\ ORCID: https://orcid.org/0000-0002-2276-0452 \\ Universidade Federal da Bahia, Brasil \\ E-mail: rosangela.jpassos@gmail.com \\ Lucivalda Pereira Magalhães de Oliveira \\ ORCID: https://orcid.org/0000-0003-4822-5930 \\ Universidade Federal da Bahia, Brasil \\ E-mail: valdapm@hotmail.com
}

\begin{abstract}
Resumo
Objetivo: avaliar o consumo alimentar habitual de pacientes com Doença Hepática Gordurosa não Alcoólica (DHGNA) de acordo com o grau de esteatose hepática, verificar a associação com variáveis antropométricas e desenvolver um Questionário de Frequência Alimentar (QFA) direcionado para essa população. Métodos: Estudo observacional de corte transversal desenvolvido com 60 pacientes adultos, diagnosticados com DHGNA leve ou moderada. Realizou-se avaliação antropométrica e aplicação de recordatórios de 24 horas. Para a análise da composição da dieta e classificação dos alimentos considerou-se a proposta NOVA. Para o desenvolvimento do QFA foram selecionados os alimentos que contribuíram com até $95 \%$ de energia, macronutrientes e micronutrientes de interesse. Utilizou-se o teste de Mann Whithey para verificar a diferença entre as medianas das variáveis com o grau de esteatose hepática e o coeficiente de correlação de Spearman para o cálculo das correlações. O nível de significância adotado na decisão dos testes foi de 5\%. Resultados: observou-se ingestão alimentar semelhante entre os pacientes com DHGNA, com predomínio dos alimentos in natura ou minimamente processados. O grau de processamento dos alimentos não apresentou correlação significativa com as medidas antropométricas e grau de esteatose. O QFA foi elaborado a partir da seleção de 96 alimentos. Conclusões: o consumo alimentar apresenta-se semelhante ao padrão básico da população brasileira. $\mathrm{O}$ consumo total de macro e micronutrientes destes pacientes não se correlacionou com a gravidade da esteatose, nem com os dados antropométricos. Espera-se que o QFA proposto torne-se uma ferramenta útil no monitoramento das práticas alimentares de indivíduos com DHGNA.
\end{abstract}

Palavras-chave: Doença hepática gordurosa não alcoólica; Consumo de alimentos; Processamento de alimentos; Inquéritos alimentares; Questionário de frequência alimentar.

\footnotetext{
Abstract

Objective: to evaluate the usual food intake of patients with Non-alcoholic Fatty Liver Disease (NAFLD) according to the degree of hepatic steatosis, to verify the association with anthropometric variables and to develop a Food Frequency Questionnaire (FFQ) targeted at this population. Methods: Observational cross-sectional study developed with 60 adult patients diagnosed with mild or moderate NAFLD. Anthropometric evaluation and 24-hour recall were performed. For the analysis of the diet composition and food classification, the NOVA proposal was considered. For the development
} 
of FFQ, foods that contributed up to $95 \%$ of energy, macronutrients and micronutrients of interest were selected. The Mann Whithey test was used to verify the difference between the medians of the variables with the grade of hepatic steatosis and the Spearman correlation coefficient for the calculation of correlations. The significance level adopted in the test decision was 5\%. Results: similar dietary intake was observed among patients with NAFLD, with predominance of natural or minimally processed foods. The degree of food processing did not present significant correlation with anthropometric measurements and steatosis degree. The FFQ was prepared from the selection of 96 foods. Conclu sions: food consumption is like the basic pattern of the Brazilian population. The total intake of macro and micronutrients of these patients did not correlate with the severity of steatosis, nor with anthropometric data. The proposed FFQ is expected to become a useful tool in monitoring dietary practices of individuals with NAFLD.

Keywords: Non-alcoholic fatty liver disease; Food consumption; Food processing; Food surveys; Food frequency questionnaire.

\section{Resumen}

Objetivo: evaluar el consumo alimentario habitual de pacientes con Enfermedad Hepática Grasa No Alcohólica (EHGNA) de acuerdo con el grado de esteatosis hepática, verificar la asociación con variables antropométricas y desarrollar un Cuestionario de Frecuencia Alimentaria (QFA) dirigido a esta población. Métodos: Estudio observacional de corte transversal desarrollado con 60 pacientes adultos, diagnosticados con EHGNA leve o moderada. Se realizó evaluación antropométrica y aplicación de recordatorios de 24 horas. Para el análisis de la composición de la dieta y clasificación de los alimentos se consideró la propuesta NOVA. Para el desarrollo del QFA se seleccionaron los alimentos que contribuyeron con hasta un $95 \%$ de energía, macronutrientes y micronutrientes de interés. Se utilizó la prueba de Mann Whithey para verificar la diferencia entre las medianas de las variables con el grado de esteatosis hepática y el coeficiente de correlación de Spearman para el cálculo de las correlaciones. El nivel de significancia adoptado en la decisión de las pruebas fue del 5\%. Resultados: se observó ingesta alimentaria similar entre los pacientes con EHGNA, con predominio de los alimentos in natura o mínimamente procesados. El grado de procesamiento de los alimentos no mostró correlación significativa con las medidas antropométricas y el grado de esteatosis. El QFA fue elaborado a partir de la selección de 96 alimentos. Conclusiones: el consumo alimentario se presenta similar al patrón básico de la población brasileña. El consumo total de macro y micronutrientes de estos pacientes no se correlacionó con la gravedad de la esteatosa, ni con los datos antropométricos. Se espera que el QFA propuesto se convierta en una herramienta útil en el monitoreo de las prácticas alimentarias de individuos con EHGNA.

Palabras clave: Enfermedad hepática grasa no alcohólica; Consumo de alimentos; Procesamiento de alimentos; Encuestas alimentarias; Cuestionario de frecuencia alimentaria.

\section{Introdução}

A Doença hepática gordurosa não alcoólica (DHGNA) é uma das doenças hepáticas crônicas mais comuns em todo o mundo, sendo considerada um grande problema de saúde pública. Caracteriza-se como uma síndrome clínica que abrange um amplo espectro de apresentações às quais podem variar desde esteatose simples até esteato-hepatite e cirrose hepática, podendo ser definida como a presença de esteatose hepática superior ou igual a 5\% dos hepatócitos, confirmada por histologia ou exame de imagem em indivíduos com um consumo diário não significativo de álcool (Chalasani et al., 2018; Younossi et al., 2019; Arab et al., 2020).

A prevalência global da DHGNA na população adulta foi estimada em aproximadamente $25 \%$ em uma meta-análise recente. O estudo incluiu 8.515.431 indivíduos provenientes de 22 países e verificou-se que 51,34\% deles apresentavam obesidade e 22,51\% diabetes (Younossi et al., 2016). A prevalência na América do Sul foi estimada em 30,4\% e apenas um estudo brasileiro, realizado em 139 indivíduos, foi incluído na análise, o qual apresentou prevalência de aproximadamente 35,2\% (Karnikowski et al., 2016).

A ocorrência de DHGNA é frequentemente associada a complicações metabólicas, portanto, há uma estimativa de aumento exponencial da doença para as próximas décadas, associado ao crescimento da epidemia global de obesidade e diabetes mellitus tipo 2 (Estes et al., 2018; Younossi et al., 2019). Recentemente foi sugerida uma nova definição da DHGNA para possibilitar a inclusão da relação desta com fatores de risco metabólicos, surgindo o termo Doença Hepática Gordurosa Associada ao Metabolismo (DHGAM), entretanto o uso da nova sigla ainda não é consenso entre os pesquisadores (Eslam et al., 2020; Mendez-Sanchez et al., 2021; Shiha et al., 2021).

Assim, devido à relação entre a DHGNA e distúrbios metabólicos, mudanças no estilo de vida e no consumo alimentar 
são fundamentais para o seu tratamento (Chalasani et al., 2018; Arab et al., 2020; Garcêz et al., 2021). Além do excesso da ingestão de calorias e a inadequação de macro e micronutrientes da dieta, a qualidade da alimentação e o consumo de alimentos processados e ultraprocessados, também podem desempenhar um papel importante na patogênese da doença, uma vez que estão associados às complicações metabólicas comuns aos pacientes com esteatose, principalmente aqueles com excesso de peso e obesidade (Rico-Campa et al., 2019; Chen et al., 2020; Mirizzi et al., 2019; Plagiai et al., 2021; Tutunchi et al., 2021).

Devido a importância da dieta na etiologia da DHGNA torna-se relevante conhecer e monitorar o consumo alimentar dos pacientes com essa enfermidade. Dentre os inquéritos de consumo mais utilizados para investigação clínica e epidemiológica, o Questionário de Frequência Alimentar (QFA) se destaca por apresentar fácil aplicabilidade, ser de baixo custo e viabilizar a obtenção de padrões alimentares habituais, permitindo a associação com condições de saúde, o que possibilita sua utilização em estudos populacionais (Fisberg et al., 2008; Fisberg et al., 2009; Pedraza \& Menezes, 2015).

Até o presente momento desconhecemos um QFA específico para indivíduos com DHGNA no Brasil e, portanto, o desenvolvimento de um instrumento adequado para auxiliar na avaliação mais fidedigna da ingestão alimentar pode ser útil para esta população. Diante do contexto apresentado, o objetivo do presente estudo foi avaliar o consumo alimentar habitual de pacientes DHGNA de acordo com o grau de esteatose hepática, verificar a associação entre ingestão alimentar com variáveis antropométricas e desenvolver um Questionário de Frequência Alimentar direcionado para essa população.

\section{Metodologia}

\subsection{Desenho e população do estudo}

Trata-se de estudo observacional de corte transversal que avaliou o consumo alimentar de 60 pacientes com idade entre 20 e 60 anos, de ambos os sexos com diagnóstico clínico de DHGNA confirmado por ultrassonografia abdominal. Esses indivíduos aceitaram participar do estudo ao comparecerem na primeira consulta com a Nutricionista no Ambulatório de Nutrição e Hepatologia do Complexo Hospitalar Universitário Professor Edgard Santos (C-HUPES), da Universidade Federal da Bahia, localizado na cidade de Salvador, Bahia.

Não foram incluídos no protocolo de pesquisa pacientes com diagnóstico de insuficiência cardíaca congestiva, doença pulmonar descompensada ou grave, neoplasias, doença renal, doenças hepáticas avançadas (Child Pugh C), esquistossomose, hemocromatose, doença de Wilson, hepatites virais ou autoimunes. Mulheres gestantes, lactantes, usuários de drogas ilícitas, pacientes com relato de ingestão de álcool superior a $20 \mathrm{~g} /$ dia para mulheres, ou 30g/dia para homens (Sanyal et al., 2011) e/ou passado de alcoolismo com abstenção inferior a seis meses, também não participaram do estudo. A existência de alguma das condições mencionadas, bem como história recente de realização de procedimento cirúrgico, de hospitalização ou infecções até 30 dias anteriormente a primeira entrevista foram consideradas capazes de alterar o consumo alimentar habitual e, portanto, foram critérios de não inclusão no estudo.

O presente estudo foi aprovado pelo Comitê de Ética e Pesquisa do Complexo Hospitalar Universitário Professor Edgard Santos (C-HUPES) (Número do Parecer: 2.635.954) e todos os pacientes elegíveis foram convidados a assinar um termo de consentimento livre e esclarecido.

\subsection{Coleta de Dados}

A coleta de dados ocorreu de julho a dezembro de 2018 e para caracterizar a população avaliada utilizou-se um questionário desenvolvido e padronizado pelos autores para coleta de informações sociodemográficas (sexo, idade, situação conjugal, renda familiar, quantidade de moradores no domicílio e escolaridade) e antropométricas (peso, altura e circunferência da cintura). Os dados clínicos, informações da ultrassonografia abdominal e presença de comorbidades foram coletados a partir dos exames mais recentes dos pacientes, considerando no máximo três meses antes da entrevista, e complementados com os 
registros em prontuário. A informação sobre o grau de esteatose hepática foi obtida a partir do exame de ultrassom de cada paciente, e para fim de análises considerou-se as categorias leve ou moderado, de acordo com a descrição do laudo médico.

A escolaridade foi registrada em anos completos de estudo formal com aprovação. A renda familiar total foi definida pelo somatório dos rendimentos de pessoas com remuneração que ocupavam o mesmo domicílio, sendo incluídos nesse valor incentivos provenientes de programas governamentais. A partir da divisão entre a renda total e a quantidade de moradores obtevese o valor de renda per capita, considerando o valor de salário-mínimo brasileiro da época.

As medidas de peso e altura foram realizadas por uma nutricionista treinada, utilizando balança eletrônica (marca Filizola ${ }^{\circledR}$ ), com a capacidade para $200 \mathrm{Kg}$ e sensibilidade de $100 \mathrm{~g}$, acoplada a uma escala métrica para a medição da estatura, com limite de 2,0 m e sensibilidade de 1,0 cm. O Índice de Massa Corporal (IMC) foi obtido pela relação entre peso (Kg) e o quadrado da altura (m). Para avaliar a circunferência da cintura (CC), utilizou-se a medida no ponto médio entre a última costela e a crista ilíaca, com o auxílio de uma fita inelástica, com escala em centímetros (Lohman et al.,1988; World Health Organization [WHO], 1998). A informação sobre o grau de esteatose hepática foi obtida a partir do exame de ultrassom de cada paciente, de acordo com o laudo médico presente, e para fim de análises considerou-se duas categorias, leve ou moderado.

Para avaliação do consumo alimentar foram realizados até três Recordatórios Alimentares de 24 horas (R24h) com cada indivíduo, um presencial no momento da primeira entrevista e dois por telefone, abrangendo um dia de final de semana (Buzzard et al., 1998; Verly Junior et al., 2013). Todas as ligações telefônicas foram realizadas por um único pesquisador previamente treinado. Durante a coleta de dados, tanto presencial como por telefone, utilizou-se os 05 passos recomendados pelo Multiple Pass Method (Moshfeg et al., 2008). e no momento da entrevista presencial utilizou-se ainda um álbum seriado com fotos de medidas caseiras e porções de alimentos produzidos para o presente projeto a partir de publicações prévias (Brasil, 1996; Monteiro et al., 2007).

\subsection{Avaliação do consumo alimentar}

Os recordatórios tiveram as medidas caseiras mencionadas para cada alimento ou preparação transformadas em gramas ou mililitros $(\mathrm{ml})$. As preparações foram desmembradas com base em receitas padronizadas, com os seus respectivos ingredientes, e convertidas em medidas caseiras, e posteriormente também transformadas em gramas $(\mathrm{g})$ ou mililitros $(\mathrm{mL})$ (Brasil, 1996; Monteiro et al., 2007; Pinheiro et al., 2005).

Após a conversão das medidas caseiras em gramas ou mililitros (ml) utilizou-se o software Virtual Nutri Plus (versão Online) para obtenção do valor energético e da composição nutricional (macro e micronutrientes) de todos os alimentos presentes nos recordatórios alimentares, de modo a obter o consumo global e individual. Foram consideradas relevantes informações do consumo alimentar referentes a ingestão de energia, carboidratos, fibra, lipídios, proteínas, ferro, zinco, selênio, vitaminas A, E, C e cálcio.

Realizou-se ainda a avaliação do perfil do consumo alimentar dos indivíduos, considerando o processamento industrial e as quantidades em gramas de cada alimento, e depois os alimentos foram classificados de acordo com o grau processamento, segundo a proposta NOVA (um nome, não acrônimo) (Monteiro et al., 2016; Monteiro et al., 2018; Monteiro et al., 2019). A classificação também é utilizada pelas atuais Diretrizes Alimentares para a População Brasileira (Brasil, 2014). A definição e descrição de cada grupo utilizada neste trabalho encontra-se no Quadro 1. 
Quadro 1. Classificação dos alimentos citados nos R24h de acordo com o grau de processamento.

\begin{tabular}{|c|c|c|}
\hline Grupo & Definição & Alimentos \\
\hline $\begin{array}{l}\text { Grupo 1: Alimentos in natura } \\
\text { ou minimamente processados }\end{array}$ & $\begin{array}{l}\text { Alimentos obtidos diretamente de } \\
\text { plantas ou de animais e adquiridos } \\
\text { para consumo sem que tenham sofrido } \\
\text { qualquer alteração após deixarem a } \\
\text { natureza ou alimentos in natura que, } \\
\text { antes de sua aquisição, foram } \\
\text { submetidos a alterações } \\
\text { mínimas. }\end{array}$ & $\begin{array}{l}\text { Frutas, legumes, raízes e tubérculos } \\
\text { frescos, refrigerados, congelados ou } \\
\text { embalados a vácuo; leguminosas e grãos } \\
\text { (cereais) secos, polidos e empacotados ou } \\
\text { moídos na forma de farinhas, sucos de } \\
\text { frutas } 100 \% \text { sem açúcar; oleaginosas e } \\
\text { sementes sem sal; carnes, aves e peixes } \\
\text { frescos, refrigerados ou congelados; leite } \\
\text { fresco, pasteurizado ou fermentado como } \\
\text { iogurte natural, ovos, chás e café. }\end{array}$ \\
\hline $\begin{array}{l}\text { Grupo 2: Ingredientes } \\
\text { culinários }\end{array}$ & $\begin{array}{l}\text { Produtos extraídos de alimentos in } \\
\text { natura ou diretamente da natureza e } \\
\text { usados pelas pessoas para temperar e } \\
\text { cozinhar alimentos e criar preparações } \\
\text { culinárias. }\end{array}$ & $\begin{array}{l}\text { Diferentes tipos de óleos e gorduras, } \\
\text { manteiga, margarina, açúcar e sal }\end{array}$ \\
\hline \multirow[t]{2}{*}{$\begin{array}{l}\text { Grupo 3: Alimentos } \\
\text { processados e } \\
\text { Ultraprocessados }\end{array}$} & $\begin{array}{l}\text { Alimentos processados: Produtos } \\
\text { fabricados essencialmente com a } \\
\text { adição de sal ou açúcar. }\end{array}$ & $\begin{array}{l}\text { Legumes em conserva, carne de charque e } \\
\text { queijos. }\end{array}$ \\
\hline & $\begin{array}{l}\text { Alimentos Ultraprocessados: Produtos } \\
\text { cuja fabricação envolve diversas } \\
\text { etapas e técnicas de processamento e } \\
\text { vários ingredientes, muitos deles de } \\
\text { uso exclusivamente industrial. }\end{array}$ & $\begin{array}{l}\text { Pães, biscoitos, bolos e pastelaria; sorvete; } \\
\text { compotas (conservas); chocolates, barras } \\
\text { de cereais, cereais matinais com adição de } \\
\text { açúcar; batatas fritas industrializadas, } \\
\text { molhos industrializados, bebidas lácteas, } \\
\text { refrigerantes, massas congeladas, carne } \\
\text { processada e embutidos }\end{array}$ \\
\hline
\end{tabular}

Fonte: Monteiro et al. (2016); Monteiro et al. (2018); Monteiro et al. (2019).

\subsection{Desenvolvimento do Questionário de Frequência Alimentar}

Seleção dos alimentos: A lista de alimentos que compôs o QFA foi elaborada a partir da análise de todos os alimentos e bebidas citados pelos pacientes nos R24h. Utilizou-se como critério de inclusão na lista deste QFA alimentos que contribuíram com até 95\% de energia, macronutrientes (carboidratos, fibras, lipídios e proteínas) e micronutrientes de interesse (ferro, zinco, selênio, vitaminas A, C, E e cálcio), segundo a metodologia proposta por Block et al. (1985). A contribuição percentual foi calculada a partir da razão entre a quantidade total do nutriente de cada alimento citado e o total deste nutriente fornecido por todos os alimentos, multiplicado por 100, como exemplificado na fórmula a seguir:

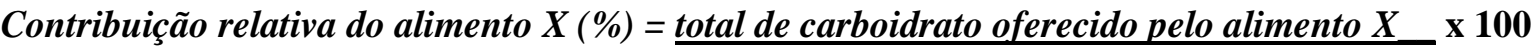 total de carboidrato oferecido por todos os alimentos}

Para que nenhum alimento considerado importante não fosse incluído no QFA, os itens que contribuíram com menos de $5 \%$ no consumo de energia e nutrientes foram revisados, no entanto não houve nenhuma outra inclusão. Os alimentos selecionados foram ordenados de modo decrescente, segundo o percentual de contribuição relativa para a oferta de energia, macronutrientes e micronutrientes de interesse. Apesar do cloreto de sódio (sal de adição) não contribuir de forma significativa para o consumo de energia ou nutrientes de interesse, optou-se por sua inclusão no QFA com o objetivo de avaliar a quantidade habitual da sua ingestão. Após a etapa de seleção, os alimentos foram organizados no QFA em grupos de acordo com o grau de processamento (Monteiro et al., 2016; Monteiro et al., 2018; Monteiro et al., 2019). 
Cálculo da porção alimentar de referência e definição dos grupos de alimentos: com a intenção de produzir um QFA quantitativo, o tamanho das porções de cada alimento foi classificado em pequeno (P), médio (M), grande (G) e extragrande (E), de acordo com a distribuição percentual dos valores equivalentes às medidas caseiras dos registros alimentares de 24 horas. A porção mediana (valor do percentil 50) foi utilizada como referência em cada item atribuindo-se aos tamanhos pequeno, grande e extragrande as quantidades apresentadas nos percentis $25\left(\mathrm{p} 25^{\circ}\right), 75\left(\mathrm{p} 75^{\circ}\right)$ e $100\left(\mathrm{p} 100^{\circ}\right)$, respectivamente. Todos os valores fracionados foram aproximados para números inteiros (Block et al., 1986; Cardoso \& Stocco et al., 2000; Sarmento et al., 2013).

Para a situação em que os valores encontrados dos percentis $\left(25^{\circ}, 50^{\circ}, 75^{\circ}\right.$ e $\left.100^{\circ}\right)$ coincidiram para um alimento, realizou-se o cálculo utilizando a regra de três simples para identificar o tamanho das porções, considerando o valor em grama ou mililitro do alimento no $\mathrm{p} 50^{\circ} \mathrm{com}$ referência (porção média) para estimar os valores das demais porções ( $\mathrm{P}, \mathrm{G}$ e $\mathrm{E}$ ) nas proporções de 25\%,75\% e 100\%, respectivamente (Fisberg et al., 2008; Berquó, 1981; Block et al., 1986). Quando foram identificadas variações das porções médias de um mesmo tipo de alimento, a exemplo das variedades de feijões, a média de cada percentil foi utilizada para determinar o tamanho de referência na porção.

Análise da frequência de consumo: Com o objetivo de avaliar quantitativamente a frequência de consumo dos alimentos listados no QFA, as unidades de tempo foram determinadas em: nunca, diário, semanal e mensal. Para cada uma dessas respostas, categorias de um a dez identificam o número de vezes que o indivíduo consome o alimento na unidade de tempo referenciada. O período mínimo de recordação foi definido como o mês anterior pois espera-se que esse curto período de recordação tenha uma boa correlação com os hábitos alimentares dos participantes (Block et al., 1985; Block et al., 1986; Jiménez \& Martínmoreno, 1995; Slater et al., 2003).

\subsection{Análise dos dados}

Para a caracterização da amostra, os dados sociodemográficos, e aqueles referentes ao valor energético e a composição nutricional foram analisados por frequências, porcentagens, medidas de tendência central e dispersão. A pressuposição de normalidade das variáveis quantitativas foi verificada por meio do teste de Shapiro-Wilk. Todos os dados referentes ao consumo alimentar tiveram a variância intrapessoal de cada alimento e nutriente corrigida por técnicas de modelagem estatística incorporadas no software Multiple Source Method (MSM) (versão 1.0.1, 2011) (Harttig et al., 2011).

Para verificar a diferença entre as medianas das variáveis sociodemográficas (idade, escolaridade, renda familiar, quantidade de moradores e renda per capta), antropométricas (peso, altura, IMC e circunferência da cintura) e as relacionadas ao consumo alimentar (energia, macronutrientes e micronutrientes) com o grau de esteatose hepática utilizou-se o teste de Mann Whithey.

Para verificar a correlação entre as variáveis antropométricas (CC e IMC) e a ingestão dos grupos alimentares, utilizouse o coeficiente de correlação de Spearman. Para essa análise realizou-se o ajuste do consumo dos nutrientes pela energia, por meio do método residual (Willett \& Stampfer, 1986). O nível de significância adotado na decisão dos testes foi de 5\%. Todas as análises, exceto o ajuste da variabilidade intrapessoal, foram realizadas no software Stata®, v.12 (Statacorp, College Station, Texas, USA).

\section{Resultados}

No presente estudo, a maioria dos pacientes avaliados era do sexo feminino (86,7\%), casada $(83,3 \%)$ e autodeclarou cor/raça parda $(75,0 \%)$. O valor da mediana para idade foi $55(48$ - 58) anos e para renda per capita $R \$ 477,0(318,0-477,0)$ ou \$ 85,1 (56,7 - 85,1), valor inferior a um salário-mínimo brasileiro na época do estudo (R $\$ 1.100$ ou \$192,2). Em relação a 
escolaridade, o valor da mediana de anos de estudo da população foi 12 (05 - 12) anos e entre os pacientes avaliados, 50\% apresentaram ensino primário ou fundamental, $45 \%$ ensino médio e apenas $5 \%$ possuíam curso superior completo.

Demais características dos pacientes e os valores da ingestão de nutrientes encontram-se descritas segundo grau de esteatose na Tabela 1. Dos 60 pacientes incluídos no estudo, 37 responderam três recordatórios, mas, por dificuldade de comunicação telefônica, 17 responderam dois R24h e seis responderam apenas um, resultando assim um total de 151 R24h. Não houve diferença significativa entre as variáveis sociodemográficas e de ingestão alimentar de acordo com o grau de esteatose ( $>0,05)$. Em relação aos dados antropométricos, observou-se maiores valores para a $\mathrm{CC}(\mathrm{p}=0,03)$ entre os pacientes com esteatose moderada (Tabela 1).

As informações sobre a ingestão de energia, macronutrientes e micronutrientes, segundo o grau de processamento dos alimentos e de esteatose dos pacientes, encontra-se descrita na Tabela 2. Observa-se que em relação aos macronutrientes houve diferença significativa para o teor de carboidratos $(\mathrm{p}=0,01)$ e lipídios totais $(\mathrm{p}=0,04)$ provenientes do Grupo 02 de ingredientes culinários, que foi mais consumido entre os pacientes com esteatose leve.

Pacientes com esteatose leve também apresentaram maior consumo de lipídios poli-insaturados $(\mathrm{p}=0,03)$ provenientes de alimentos in natura ou minimamente processados (Grupo 1). Conforme esperado, para esses pacientes a densidade calórica da dieta foi aproximadamente duas vezes menor para os alimentos in natura e minimamente processados (Grupo 1), quando comparado aos alimentos processados e ultraprocessados (Grupo 3) (Tabela 2).

No que diz respeito aos micronutrientes, a diferença da ingestão entre os grupos foi estatisticamente significante para a vitamina A e cálcio, provenientes dos alimentos in natura ou minimamente processados (Grupo 1) e processados ou ultraprocessados (Grupo 3), respetivamente. Observou-se maior ingestão de Vitamina A ( $\mathrm{p}=0,01)$ entre os indivíduos com esteatose moderada, e maior ingestão de cálcio no grupo com esteatose leve ( $\mathrm{p}=0,01)$ (Tabela 2).

Verificou-se que os alimentos mais citados nos R24h dos pacientes com DHGNA são similares aqueles geralmente consumidos pela população brasileira. As Figuras 1a, 1b e 1c apresentam um resumo destes alimentos conforme grau de processamento. Porém, não houve correlação significativa entre o consumo alimentar e os valores de CC ou IMC (Tabela 3). 
Tabela 1. Características sociodemográficas, antropométricas e ingestão alimentar dos pacientes com DHGNA, avaliada de acordo com o grau de esteatose hepática.

\begin{tabular}{|c|c|c|c|c|}
\hline & $\begin{array}{c}\begin{array}{c}\text { Total } \\
(\mathbf{n}=60)\end{array} \\
\end{array}$ & $\begin{array}{c}\begin{array}{c}\text { Esteatose leve } \\
(\mathrm{n}=\mathbf{2 4})\end{array} \\
\end{array}$ & Esteatose moderada $(\mathrm{n}=\mathbf{3 6})$ & $p$-valor ${ }^{\#}$ \\
\hline Idade (anos) & $55,0(48,0-58,0)$ & $54,5(49,5-58,0)$ & $55,0(46,5-55,0)$ & 0,83 \\
\hline $\begin{array}{c}\text { Escolaridade } \\
\text { (anos de estudo) }\end{array}$ & $12(05-12)$ & $13(6,5-14)$ & $09(5,5-13)$ & 0,21 \\
\hline Renda familiar total $(\mathrm{R} \$)$ & $1.217,0(954-1904)$ & $1.292,5(954-1894)$ & $1.117,0(954,0-1904,0)$ & 0,78 \\
\hline Quantidade de moradores & $03(02-04)$ & $03(02-04)$ & $03(02-04)$ & 0,9 \\
\hline Renda per capta $(\mathrm{R} \$)$ & $477,0(318,0-477,0)$ & $500,0(327,6-952)$ & $477,0(318,0-715,5)$ & 0,36 \\
\hline Peso $(\mathrm{Kg})$ & $85,2(73,8-97,4)$ & $82,1(70,4-88,3)$ & $88,3(75,9-101,1)$ & 0,13 \\
\hline Altura (m) & $1,56(1,50-1,60)$ & $1,57(1,51-1,61)$ & $1,56(1,51-1,60)$ & 0,80 \\
\hline $\operatorname{IMC}\left(\mathrm{Kg} / \mathrm{m}^{2}\right)$ & $33,4(30,5-38,9)$ & $32,0(29,0-36,1)$ & $35,5(31,6-41,2)$ & 0,06 \\
\hline $\mathrm{CC}(\mathrm{cm})$ & $106(99,7-117,5)$ & $103,5(96-110)$ & $109,5(101,5-120)$ & $\mathbf{0 , 0 3 *}$ \\
\hline Energia (Kcal) & $1225,6(1087,7-1362,8)$ & $1221,3(1091,1-1455,1)$ & $1225,6(1078,8-1344,9)$ & 0,84 \\
\hline Carboidrato (g) & $185,2(151,8-212,0)$ & $182,9(163,3-211,4)$ & $186,8(142,7-212,8)$ & 0,57 \\
\hline Fibra (g) & $18,5(14,9-20,7)$ & $19,6(17,4-20,9)$ & $17,6(13,0-20,3)$ & 0,10 \\
\hline Lipídio (g) & $35,0(25,5-42,6)$ & $32,1(21,7-37,7)$ & $35,3(26,0-50,5)$ & 0,23 \\
\hline Proteína (g) & $65,4(55,2-75,3)$ & $63,6(51,2-76,6)$ & $65,9(55,8-75,0)$ & 0,71 \\
\hline Proteína $(\mathrm{g} / \mathrm{Kg})$ & $0,7(0,6-1,0)$ & $0,7(0,5-0,8)$ & $0,8(0,6-1,0)$ & 0,37 \\
\hline Vitamina A ( $\mu \mathrm{g}$ RE) & $1.359,3(856,8-1684,9)$ & $1.422,9(1174,0-1738,2)$ & $1.205,7(770,6-1634,2)$ & 0,19 \\
\hline Vitamina C (mg) & $90,1(50,0-152,5)$ & $91,9(60,6-175,9)$ & $88,7(46,4-147,9)$ & 0,51 \\
\hline Vitamina E (mg) & $6,6(3,5-9,5)$ & $7,0(4,8-9,5)$ & $6,6(2,9-9,5)$ & 0,66 \\
\hline Cálcio (mg) & $447,4(305,5-627,7)$ & $460,6(369,5-610,9)$ & $427,5(295,4-646,8)$ & 0,63 \\
\hline Ferro (mg) & $9,7(5,9-12,4)$ & $10,2(7,0-12,5)$ & $8,8(5,0-12,2)$ & 0,45 \\
\hline Selênio (mg) & $31,6(22,3-57,6)$ & $31,3(21,4-60,0)$ & $34,1(22,3-57,2)$ & 0,56 \\
\hline Zinco (mg) & $5,7(1,2-6,9)$ & $5,8(3,7-6,8)$ & $5,6(1,1-7,0)$ & 0,80 \\
\hline
\end{tabular}

Resultados expressos como mediana e interquartis (amplitude $25-75 \%$ ); R $\$=$ real; $\mathrm{Kg}=$ Quilograma; $\mathrm{m}=$ metro; IMC = Índice de Massa Corporal; $\mathrm{CC}=$ circunferência da cintura; $\mathrm{cm}=$ centímetro; Kcal $=$ Quilocaloria; $\mathrm{g}=$ grama; $\mathrm{mg}=$ miligrama; $\mu \mathrm{g} \mathrm{RE}=$ microgramas de retinol; $-=$ quantidade não relevante do nutriente (próxima de zero); ${ }^{*}$ teste de Mann Whithey; *diferença estatisticamente significativa. Fonte: Autores (2021). 
Research, Society and Development, v. 10, n. 16, e280101623506, 2021

(CC BY 4.0) | ISSN 2525-3409 | DOI: http://dx.doi.org/10.33448/rsd-v10i16.23506

Tabela 2. Consumo de energia, macronutrientes e micronutrientes dos pacientes com DHGNA, segundo o grupo alimentar e grau de esteatose hepática

\begin{tabular}{|c|c|c|c|c|c|c|c|c|c|}
\hline & Grupo 1 & $\begin{array}{r}\text { Esteatose Leve } \\
\text { Grupo } 2\end{array}$ & Gruno 3 & & Esteatose Moderada & & & $n$-valor ${ }^{\#} \mathbf{G} 2$ & $p$-valor" $\mathbf{G 3}$ \\
\hline Quantidade (g) & $\begin{array}{c}947,7 \\
(794,8-1050,5)\end{array}$ & $\begin{array}{c}17,6 \\
(13,1-20,6)\end{array}$ & $\begin{array}{c}92,6 \\
(67,8-146,1)\end{array}$ & $\begin{array}{c}987,3 \\
(841,9-1149,5)\end{array}$ & $\begin{array}{c}18,7 \\
(14,9-25,1)\end{array}$ & $\begin{array}{c}81,0 \\
(46,6-128,8)\end{array}$ & 0,52 & 0,40 & 0,30 \\
\hline Energia (Kcal) & $\begin{array}{c}861,8 \\
(731,4-928,6)\end{array}$ & $\begin{array}{c}95,7 \\
(94,1-105,6)\end{array}$ & $\begin{array}{c}229,7 \\
(198,5-303,4)\end{array}$ & $\begin{array}{c}876,5 \\
(764,5-1049,4)\end{array}$ & $\begin{array}{c}95,7 \\
(94,1-104,3)\end{array}$ & $\begin{array}{c}191,8 \\
(130,7-320,2)\end{array}$ & 0,48 & 0,95 & 0,17 \\
\hline $\begin{array}{c}\text { Densidade calórica do grupo } \\
(\mathrm{Kcal} / \mathrm{g})\end{array}$ & $0,9(0,8-1,0)$ & $5,4(4,6-7,7)$ & $2,25(1,9-3,1)$ & $0,9(0,8-1,0)$ & $4,6(3,8-6,4)$ & $2,5(1,2-2,9)$ & 0,37 & 0,10 & 0,88 \\
\hline Carboidrato $(\mathrm{g})$ & $\begin{array}{c}124,1 \\
(95,1-157,3)\end{array}$ & $\begin{array}{c}10,8 \\
(7,6-16,2)\end{array}$ & $\begin{array}{c}42,6 \\
(28,5-54,7)\end{array}$ & $\begin{array}{c}142,8 \\
(97,0-159,4)\end{array}$ & $\begin{array}{c}4,8 \\
(1,4-11,7)\end{array}$ & $\begin{array}{c}35,5 \\
(26,4-50,6)\end{array}$ & 0,54 & $0,01 *$ & 0,42 \\
\hline Fibras (g) & $\begin{array}{c}15,7 \\
(11,6-18,8)\end{array}$ & - & $2,2(1,9-2,3)$ & $16,8(13,3-18,9)$ & - & $2,2(2,1-2,4)$ & 0,67 & - & 0,65 \\
\hline Lipídios totais (g) & $\begin{array}{c}19,6 \\
(12,7-23,5)\end{array}$ & $8,1(6,7-11,0)$ & $9,2(5,6-17,5)$ & $18,7(9,4-20,9)$ & $7,2(1,1-9,0)$ & $6,7(4,1-9,9)$ & 0,48 & $0,04 *$ & 0,06 \\
\hline Lipídios saturados (g) & $\begin{array}{c}5,4 \\
(3,4-7,3)\end{array}$ & $1,3(0,0-1,5)$ & $1,8(1,2-2,7)$ & $6,9(4,4-10,0)$ & $0,1-(0,0-1,3)$ & $1,3(0,0-2,1)$ & 0,18 & 0,14 & 0,06 \\
\hline Lipídios monoinsaturados (g) & $\begin{array}{c}5,7 \\
(3,9-7,6)\end{array}$ & $1,7(0,0-2,2)$ & - & $5,2(3,1-6,7)$ & $0,1(0,0-2,1)$ & - & 0,35 & 0,15 & - \\
\hline Lipídios poli-insaturados (g) & $\begin{array}{c}3,6 \\
(3,1-4,8)\end{array}$ & $3,8(0,0-4,7)$ & - & $2,9(2,1-4,1)$ & $1,5(0,0-3,8)$ & - & $0,03 *$ & 0,07 & - \\
\hline Proteínas (g) & $\begin{array}{c}61,6 \\
(49,1-72,8)\end{array}$ & - & $7,4(4,7-12,8)$ & $54,9(42,0-61,2)$ & - & $7,5(4,9-10,7)$ & 0,09 & - & 0,97 \\
\hline Vitamina A ( $\mu \mathrm{g}$ RE) & $\begin{array}{c}1.043,8 \\
(465,3-1290,0)\end{array}$ & $3,7(0,7-8,9)$ & - & $\begin{array}{c}1.443,0(1102,5- \\
1682,1)\end{array}$ & $2,2(0,0-5,9)$ & - & $0,01 *$ & 0,33 & - \\
\hline Vitamina C (mg) & $89,2(44,1-156,3)$ & - & - & $90,3(53,4-152,5)$ & - & - & 0,8 & - & - \\
\hline Vitamina E (mg) & $3,4(2,1-4,8)$ & $4,9(0,6-6,8)$ & - & $2,6(0,0-4,0)$ & $4,4(0,0-6,1)$ & - & 0,07 & 0,26 & - \\
\hline Cálcio (mg) & $332,1(220,0-499,7)$ & - & $149,7(88,6-315,9)$ & $297,5(204,9-406,7)$ & - & $71,2(52,7-122,8)$ & 0,33 & - & $0,01 *$ \\
\hline Ferro (mg) & $6,1(3,4-7,7)$ & - & $4,1(1,2-14,1)$ & $6,7(3,2-7,8)$ & - & $2,7(0,0-4,9)$ & 0,78 & - & 0,10 \\
\hline Selênio (mg) & $26,4(18,8-35,8)$ & - & $1,65(0,0-12,3)$ & $23,6(16,3-40,8)$ & - & $1,25(0,0-17,9)$ & 0,41 & - & 0,68 \\
\hline Zinco (mg) & $5,9(4,2-8,1)$ & - & - & $5,4(1,1-6,7)$ & - & - & 0,27 & - & - \\
\hline
\end{tabular}

Resultados expressos como mediana e interquartis (amplitude 25 - 75\%); - = quantidade não relevante do nutriente (próxima de zero); "teste de Mann Whithey; *diferença estatisticamente significativa; Grupo 1:

Alimentos in natura ou minimamente processados; Grupo 2: Ingredientes culinários; Grupo 3: Alimentos processados e Ultraprocessados; $\mu \mathrm{g}$ RE $=$ microgramas de retinol; mg = miligrama; $\mu \mathrm{g}$ RE = microgramas de retinol. Fonte: Autores (2021). 
Figura 1. Frequência dos alimentos mais citados pelos pacientes com DHGNA, segundo grau de processamento. Figura 1a (Grupo 1); 1b (Grupo 2); 1c (Grupo 3).

$1 \mathbf{a}$

GRUPO A: ALIMENTOS IN NATURA OU MINIMAMENTE PROCESSADOS

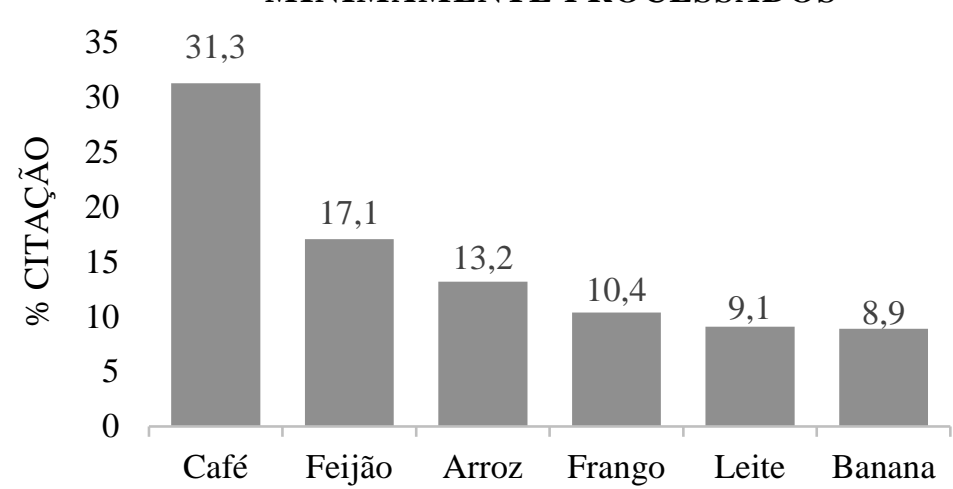

$1 \mathbf{b}$

\section{GRUPO B: INGREDIENTES CULINÁRIOS}

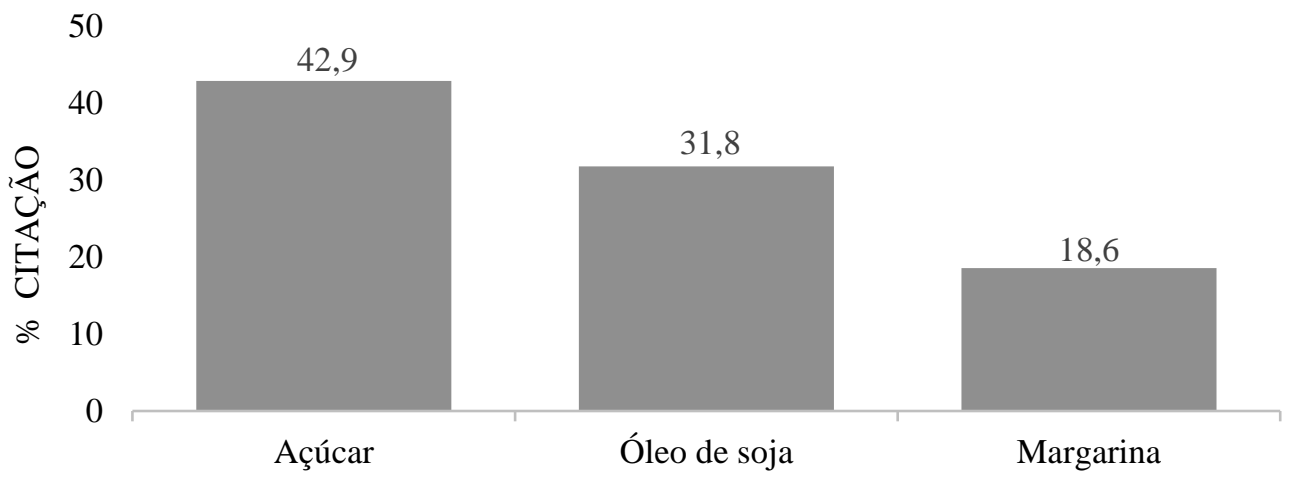

1c GRUPO C: ALIMENTOS PROCESSADOS E

ULTRAPROCESSADOS

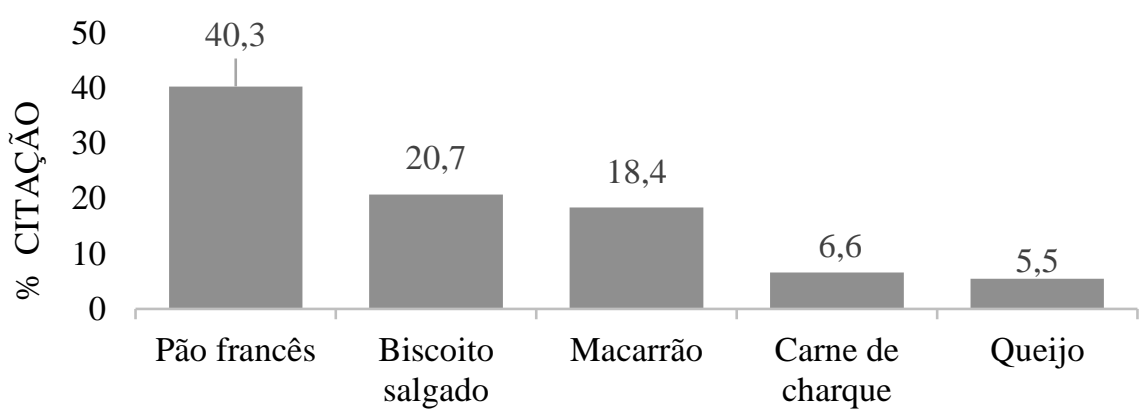

Fonte: Autores (2021) 
Research, Society and Development, v. 10, n. 16, e280101623506, 2021

(CC BY 4.0) | ISSN 2525-3409 | DOI: http://dx.doi.org/10.33448/rsd-v10i16.23506

Tabela 3 - Coeficiente de correlação de Spearman dos valores de CC e IMC dos pacientes com DHGNA e consumo dos grupos alimentares.

\begin{tabular}{|c|c|c|c|c|c|c|c|c|c|c|c|c|}
\hline & \multicolumn{6}{|c|}{$\mathrm{CC}$} & \multicolumn{6}{|c|}{ IMC } \\
\hline & \multicolumn{2}{|c|}{ Grupo 1} & \multicolumn{2}{|c|}{ Grupo 2} & \multicolumn{2}{|c|}{ Grupo 3} & \multicolumn{2}{|c|}{ Grupo 1} & \multicolumn{2}{|c|}{ Grupo 2} & \multicolumn{2}{|c|}{ Grupo 3} \\
\hline & $\rho$ & p-valor & $\rho$ & p-valor & $\rho$ & p-valor & $\rho$ & p-valor & $\rho$ & p-valor & $\rho$ & p-valor \\
\hline Quantidade (g) & $-0,10$ & 0,44 & 0,05 & 0,64 & 0,03 & 0,79 & $-0,10$ & 0,41 & $-0,08$ & 0,52 & $-0,01$ & 0,98 \\
\hline Energia (Kcal) & 0,12 & 0,33 & -0.13 & 0,31 & $-0,01$ & 0,90 & 0,08 & 0,53 & $-0,17$ & 0,18 & $-0,01$ & 0,90 \\
\hline Carboidrato (g) & $-0,10$ & 0,45 & $-0,01$ & 0,47 & 0,10 & 0,42 & $-0,18$ & 0,16 & $-0,15$ & 0,25 & 0,04 & 0,73 \\
\hline Fibras (g) & 0,01 & 0,94 & - & - & 0,02 & 0,86 & $-0,04$ & 0,73 & - & - & 0,01 & 0,97 \\
\hline Lipídios totais (g) & $-0,01$ & 0,54 & $-0,05$ & 0,67 & $-0,11$ & 0,38 & $-0,09$ & 0,44 & $-0,08$ & 0,50 & $-0,01$ & 0,99 \\
\hline Lipídios saturados (g) & 0,18 & 0,16 & 0,11 & 0,40 & $-0,01$ & 0,92 & 0,12 & 0,33 & 0,05 & 0,70 & $-0,01$ & 0,91 \\
\hline $\begin{array}{l}\text { Lipídios monoinsaturados } \\
\text { (g) }\end{array}$ & 0,08 & 0,52 & 0,06 & 0,61 & $-0,03$ & 0,77 & 0,01 & 0,98 & $-0,01$ & 0,90 & $-0,14$ & 0,27 \\
\hline Lipídios poli-insaturados & $-0,01$ & 0,89 & 0,05 & 0,66 & $-0,01$ & 0,95 & $-0,06$ & 0,61 & 0,01 & 0,96 & $-0,11$ & 0,39 \\
\hline Proteínas $(\mathrm{g})$ & $-0,01$ & 0,90 & - & - & 0,17 & 0,18 & $-0,02$ & 0,82 & - & - & 0,20 & 0,10 \\
\hline
\end{tabular}

$\rho=$ Valor do coeficiente de correlação de Spearman; - = quantidade não relevante do nutriente (próxima de zero); Grupo 1: Alimentos in natura ou minimamente processados; Grupo 2: Ingredientes culinários; Grupo 3: Alimentos processados e Ultraprocessados; Kcal = Quilocaloria; g = grama.

Fonte: Autores (2021). 
A avaliação do consumo alimentar habitual, a partir dos 151 R24h, identificou que 96 alimentos contribuíam em 95\% ou mais na ingestão da energia total, macronutrientes e micronutrientes de interesse, e, portanto, foram selecionados para compor o QFA proposto neste estudo (Figura 2). Dos alimentos que compõem o instrumento, 62,5\% são in natura ou minimamente processados, 7,3\% ingredientes culinários, 3,1\% processados e 27,1\% ultraprocessados. A versão final do QFA proposto incluiu também perguntas adicionais para investigação do uso de suplementos alimentares, possível consumo de algum alimento não presente na lista apresentada e consumo de gorduras visíveis das carnes e peles de frango ou peixe. O QFA completo e a tabela com a definição das porções para cada alimento, em gramas ou mililitros, assim como sua respectiva medida caseira, estão disponíveis na Figura 2 e na Tabela 4 deste manuscrito.

Figura 2. Questionário de Frequência Alimentar

Público: pessoas maiores a 20 anos e com Doença Hepática Gordurosa Não Alcoólica (DHGNA)

Data da entrevista:

/

\section{Número do questionário:}

\section{Nome do entrevistador:}

No do prontuário:

Nome do paciente:

1. Você está tomando algo para suplementar sua alimentação? (vitaminas, minerais e/ou outros produtos)

(1) não (2) sim

2. Se a resposta da pergunta anterior for sim, favor preencher o quadro abaixo:

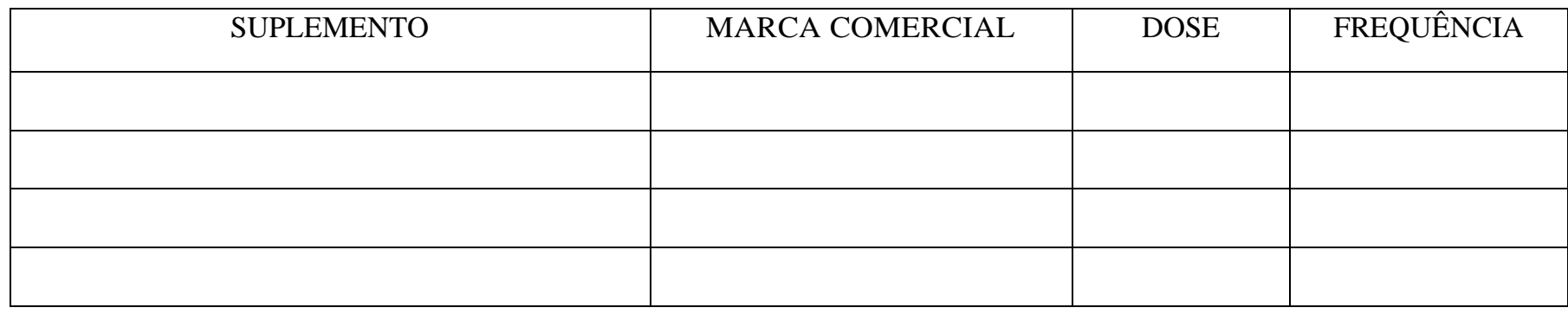

As questões seguintes relacionam-se ao seu hábito alimentar. Para cada quadro, responda, por favor, a frequência que melhor descreva QUANTAS VEZES você costuma comer cada item e a respectiva UNIDADE DE TEMPO (se por dia, por semana ou por mês). Depois, responda qual a sua PORÇÃO INDIVIDUAL USUAL em relação à porção média indicada. ESCOLHA SOMENTE UM QUADRADO para cada coluna. Se você não come ou raramente come determinado item, preencha o QUADRADO da primeira coluna ( $\mathrm{N}=$ nunca ou raramente come). NÃO DEIXE ITENS EM BRANCO. 


\begin{tabular}{|c|c|c|c|c|c|c|c|c|c|c|c|c|c|c|c|c|c|c|c|}
\hline \multirow{4}{*}{$\begin{array}{c}\text { Alimentos } \\
\\
\\
\text { Grupo 1: } \\
\text { Alimentos in } \\
\text { natura ou } \\
\text { minimamente } \\
\text { processados }\end{array}$} & \multicolumn{11}{|c|}{$\begin{array}{l}\text { Frequência de consumo } \\
\text { cê costuma comer? }\end{array}$} & \multicolumn{3}{|c|}{ Unidade } & \multicolumn{5}{|c|}{$\begin{array}{l}\text { Qual o tamanho da sua porção } \\
\text { em relação à porção média? }\end{array}$} \\
\hline & \multirow{2}{*}{\multicolumn{11}{|c|}{$\begin{array}{l}\text { Número de vezes: } 1,2,3 \text { etc. ( } \mathrm{N}=\text { nunca ou } \\
\text { raramente comeu no mês). } \\
\text { Observação: quando houver a opção de mais de } \\
\text { um tipo do alimento, relate aquele que você } \\
\text { consome com mais frequência. }\end{array}$}} & \multicolumn{3}{|c|}{$\begin{array}{c}\mathrm{D}=\mathrm{dia} \\
\mathrm{S}=\text { semana } \\
\mathrm{M}=\text { mês }\end{array}$} & $\begin{array}{l}\text { Porção média } \\
(\mathbf{M})=\text { Porção } \\
\text { de Referência }\end{array}$ & \multicolumn{4}{|c|}{$\begin{array}{c}\mathbf{P}=\text { menor que a } \\
\text { porção média } \\
\mathbf{M}=\text { igual à } \\
\text { porção média } \\
\mathbf{G}=\text { maior que a } \\
\text { porção média } \\
\mathbf{E}=\text { bem maior } \\
\text { que a porção } \\
\text { média (extra } \\
\text { grande) }\end{array}$} \\
\hline & & & & & & & & & & & & \multicolumn{3}{|c|}{ Unidade } & Porção média & \multicolumn{4}{|c|}{ Sua porção } \\
\hline & $\mathrm{N}$ & 1 & 2 & 3 & 4 & 5 & 6 & 7 & 8 & 9 & 10 & $\mathrm{D}$ & $\mathrm{S}$ & M & M & $\mathrm{P}$ & M & G & $\mathrm{E}$ \\
\hline Abóbora & $\square$ & $\square$ & $\square$ & $\square$ & $\square$ & $\square$ & $\square$ & $\square$ & $\square$ & $\square$ & $\square$ & $\square$ & $\square$ & $\square$ & $\begin{array}{c}\text { Duas } \\
\text { colheres de } \\
\text { sopa cheias } \\
(72 \mathrm{~g})\end{array}$ & $\square$ & $\square$ & $\square$ & $\square$ \\
\hline Abobrinha & $\square$ & $\square$ & $\square$ & $\square$ & $\square$ & $\square$ & $\square$ & $\square$ & $\square$ & $\square$ & $\square$ & $\square$ & $\square$ & $\square$ & $\begin{array}{c}\text { Três colheres } \\
\text { de sopa } \\
\text { cheias }(90 \mathrm{~g})\end{array}$ & $\square$ & $\square$ & $\square$ & $\square$ \\
\hline Acelga & $\square$ & $\square$ & $\square$ & $\square$ & $\square$ & $\square$ & $\square$ & $\square$ & $\square$ & $\square$ & $\square$ & $\square$ & $\square$ & $\square$ & $\begin{array}{c}\text { Três colheres } \\
\text { de sopa } \\
\text { cheias }(18 \mathrm{~g})\end{array}$ & $\square$ & $\square$ & $\square$ & $\square$ \\
\hline Alface & $\square$ & $\square$ & $\square$ & $\square$ & $\square$ & $\square$ & $\square$ & $\square$ & $\square$ & $\square$ & $\square$ & $\square$ & $\square$ & $\square$ & $\begin{array}{l}\text { Duas folhas } \\
\text { grandes }(30 \mathrm{~g})\end{array}$ & $\square$ & $\square$ & $\square$ & $\square$ \\
\hline Rúcula & $\square$ & $\square$ & $\square$ & $\square$ & $\square$ & $\square$ & $\square$ & $\square$ & $\square$ & $\square$ & $\square$ & $\square$ & $\square$ & $\square$ & $\begin{array}{c}\text { Quatro } \\
\text { colheres de } \\
\text { sopa cheias } \\
(32 \mathrm{~g})\end{array}$ & $\square$ & $\square$ & $\square$ & $\square$ \\
\hline Couve & $\square$ & $\square$ & $\square$ & $\square$ & $\square$ & $\square$ & $\square$ & $\square$ & $\square$ & $\square$ & $\square$ & $\square$ & $\square$ & $\square$ & $\begin{array}{l}\text { Duas folhas } \\
\text { médias }(40 \mathrm{~g})\end{array}$ & $\square$ & $\square$ & $\square$ & $\square$ \\
\hline Repolho & $\square$ & $\square$ & $\square$ & $\square$ & $\square$ & $\square$ & $\square$ & $\square$ & $\square$ & $\square$ & $\square$ & $\square$ & $\square$ & $\square$ & $\begin{array}{c}\text { Três colheres } \\
\text { de sopa } \\
\text { cheias }(30 \mathrm{~g})\end{array}$ & $\square$ & $\square$ & $\square$ & $\square$ \\
\hline Couve-flor & $\square$ & $\square$ & $\square$ & $\square$ & $\square$ & $\square$ & $\square$ & $\square$ & $\square$ & $\square$ & $\square$ & $\square$ & $\square$ & $\square$ & $\begin{array}{c}\text { Três ramos } \\
\text { pequenos } \\
(90 \mathrm{~g})\end{array}$ & $\square$ & $\square$ & $\square$ & $\square$ \\
\hline Cebola & $\square$ & $\square$ & $\square$ & $\square$ & $\square$ & $\square$ & $\square$ & $\square$ & $\square$ & $\square$ & $\square$ & $\square$ & $\square$ & $\square$ & $\begin{array}{c}\text { Duas } \\
\text { colheres de } \\
\text { sopa cheias } \\
(20 \mathrm{~g})\end{array}$ & $\square$ & $\square$ & $\square$ & $\square$ \\
\hline Chuchu & $\square$ & $\square$ & $\square$ & $\square$ & $\square$ & $\square$ & $\square$ & $\square$ & $\square$ & $\square$ & $\square$ & $\square$ & $\square$ & $\square$ & $\begin{array}{c}\text { Duas } \\
\text { colheres de } \\
\text { sopa cheias } \\
(40 \mathrm{~g})\end{array}$ & $\square$ & $\square$ & $\square$ & $\square$ \\
\hline Pepino & $\square$ & $\square$ & $\square$ & $\square$ & $\square$ & $\square$ & $\square$ & $\square$ & $\square$ & $\square$ & $\square$ & $\square$ & $\square$ & $\square$ & $\begin{array}{c}\text { Duas } \\
\text { colheres de } \\
\text { sopa cheias } \\
(26 \mathrm{~g})\end{array}$ & $\square$ & $\square$ & $\square$ & $\square$ \\
\hline Jiló & $\square$ & $\square$ & $\square$ & $\square$ & $\square$ & $\square$ & $\square$ & $\square$ & $\square$ & $\square$ & $\square$ & $\square$ & $\square$ & $\square$ & $\begin{array}{l}\text { Uma unidade } \\
\text { média }(39 \mathrm{~g})\end{array}$ & $\square$ & $\square$ & $\square$ & $\square$ \\
\hline Tomate & $\square$ & $\square$ & $\square$ & $\square$ & $\square$ & $\square$ & $\square$ & $\square$ & $\square$ & $\square$ & $\square$ & $\square$ & $\square$ & $\square$ & $\begin{array}{c}\text { Uma unidade } \\
\text { pequena } \\
(60 \mathrm{~g})\end{array}$ & $\square$ & $\square$ & $\square$ & $\square$ \\
\hline
\end{tabular}




\begin{tabular}{|c|c|c|c|c|c|c|c|c|c|c|c|c|c|c|c|c|c|c|c|}
\hline Maxixe & $\square$ & $\square$ & $\square$ & $\square$ & $\square$ & $\square$ & $\square$ & $\square$ & $\square$ & $\square$ & $\square$ & $\square$ & $\square$ & $\square$ & $\begin{array}{c}\text { Duas } \\
\text { unidades } \\
\text { grandes }(40 \mathrm{~g})\end{array}$ & $\square$ & $\square$ & $\square$ & $\square$ \\
\hline Quiabo & $\square$ & $\square$ & $\square$ & $\square$ & $\square$ & $\square$ & $\square$ & $\square$ & $\square$ & $\square$ & $\square$ & $\square$ & $\square$ & $\square$ & $\begin{array}{c}\text { Duas } \\
\text { unidades } \\
\text { grandes }(40 \mathrm{~g})\end{array}$ & $\square$ & $\square$ & $\square$ & $\square$ \\
\hline Vagem & $\square$ & $\square$ & $\square$ & $\square$ & $\square$ & $\square$ & $\square$ & $\square$ & $\square$ & $\square$ & $\square$ & $\square$ & $\square$ & $\square$ & $\begin{array}{c}\text { Quatro } \\
\text { colheres de } \\
\text { sopa cheias } \\
(80 \mathrm{~g})\end{array}$ & $\square$ & $\square$ & $\square$ & $\square$ \\
\hline Beterraba & $\square$ & $\square$ & $\square$ & $\square$ & $\square$ & $\square$ & $\square$ & $\square$ & $\square$ & 口 & $\square$ & $\square$ & $\square$ & $\square$ & $\begin{array}{c}\text { Duas } \\
\text { colheres de } \\
\text { sopa cheias } \\
(40 \mathrm{~g}) \\
\end{array}$ & $\square$ & $\square$ & $\square$ & $\square$ \\
\hline Cenoura & $\square$ & $\square$ & $\square$ & $\square$ & $\square$ & $\square$ & $\square$ & $\square$ & $\square$ & $\square$ & $\square$ & $\square$ & $\square$ & $\square$ & $\begin{array}{c}\text { Duas } \\
\text { colheres de } \\
\text { sopa cheias } \\
(50 \mathrm{~g})\end{array}$ & $\square$ & $\square$ & $\square$ & $\square$ \\
\hline $\begin{array}{c}\text { Banana da terra } \\
\text { cozida }\end{array}$ & $\square$ & $\square$ & $\square$ & $\square$ & $\square$ & $\square$ & $\square$ & $\square$ & $\square$ & $\square$ & $\square$ & $\square$ & $\square$ & $\square$ & $\begin{array}{c}\text { Uma unidade } \\
\text { pequena } \\
(107 \mathrm{~g})\end{array}$ & $\square$ & $\square$ & $\square$ & $\square$ \\
\hline Inhame cozido & $\square$ & $\square$ & $\square$ & $\square$ & $\square$ & $\square$ & $\square$ & $\square$ & $\square$ & $\square$ & $\square$ & $\square$ & $\square$ & $\square$ & $\begin{array}{c}\text { Duas fatias } \\
\text { médias } \\
(172 \mathrm{~g})\end{array}$ & $\square$ & $\square$ & $\square$ & $\square$ \\
\hline Aipim cozido & $\square$ & $\square$ & $\square$ & $\square$ & $\square$ & $\square$ & $\square$ & $\square$ & $\square$ & $\square$ & $\square$ & $\square$ & $\square$ & $\square$ & $\begin{array}{l}\text { Dois pedaços } \\
\text { médios } \\
(200 \mathrm{~g})\end{array}$ & $\square$ & $\square$ & $\square$ & $\square$ \\
\hline Batata doce cozida & $\square$ & $\square$ & $\square$ & $\square$ & $\square$ & $\square$ & $\square$ & $\square$ & $\square$ & $\square$ & $\square$ & $\square$ & $\square$ & $\square$ & $\begin{array}{l}\text { Dois pedaços } \\
\text { médios } \\
(140 \mathrm{~g})\end{array}$ & $\square$ & $\square$ & $\square$ & $\square$ \\
\hline $\begin{array}{l}\text { Batata inglesa } \\
\text { cozida }\end{array}$ & $\square$ & $\square$ & $\square$ & $\square$ & $\square$ & $\square$ & $\square$ & $\square$ & $\square$ & $\square$ & $\square$ & $\square$ & $\square$ & $\square$ & $\begin{array}{c}\text { Duas } \\
\text { colheres de } \\
\text { sopa cheias } \\
(60 \mathrm{~g})\end{array}$ & $\square$ & $\square$ & $\square$ & $\square$ \\
\hline Banana & $\square$ & $\square$ & $\square$ & $\square$ & $\square$ & $\square$ & $\square$ & $\square$ & $\square$ & $\square$ & $\square$ & $\square$ & $\square$ & $\square$ & $\begin{array}{l}\text { Uma unidade } \\
\text { média }(60 \mathrm{~g})\end{array}$ & $\square$ & $\square$ & $\square$ & $\square$ \\
\hline Abacaxi & $\square$ & $\square$ & $\square$ & $\square$ & $\square$ & $\square$ & $\square$ & $\square$ & $\square$ & $\square$ & $\square$ & $\square$ & $\square$ & $\square$ & $\begin{array}{c}\text { Duas rodelas } \\
\text { médias } \\
(178 \mathrm{~g})\end{array}$ & $\square$ & $\square$ & $\square$ & $\square$ \\
\hline Goiaba & $\square$ & $\square$ & $\square$ & $\square$ & $\square$ & $\square$ & $\square$ & $\square$ & $\square$ & $\square$ & $\square$ & $\square$ & $\square$ & $\square$ & $\begin{array}{l}\text { Uma unidade } \\
\text { média }(170 \mathrm{~g})\end{array}$ & $\square$ & $\square$ & $\square$ & $\square$ \\
\hline Maçã & $\square$ & $\square$ & $\square$ & $\square$ & $\square$ & $\square$ & $\square$ & $\square$ & $\square$ & $\square$ & $\square$ & $\square$ & $\square$ & $\square$ & $\begin{array}{l}\text { Uma unidade } \\
\text { média }(130 \mathrm{~g})\end{array}$ & $\square$ & $\square$ & $\square$ & $\square$ \\
\hline Laranja & $\square$ & $\square$ & $\square$ & $\square$ & $\square$ & $\square$ & $\square$ & $\square$ & $\square$ & $\square$ & $\square$ & $\square$ & $\square$ & $\square$ & $\begin{array}{l}\text { Uma unidade } \\
\text { média }(170 \mathrm{~g})\end{array}$ & $\square$ & $\square$ & $\square$ & $\square$ \\
\hline Mamão & $\square$ & $\square$ & $\square$ & $\square$ & $\square$ & $\square$ & $\square$ & $\square$ & $\square$ & $\square$ & $\square$ & $\square$ & $\square$ & $\square$ & $\begin{array}{c}\text { Uma fatia } \\
\text { média }(113 \mathrm{~g})\end{array}$ & $\square$ & $\square$ & $\square$ & $\square$ \\
\hline Manga & $\square$ & $\square$ & $\square$ & $\square$ & $\square$ & $\square$ & $\square$ & $\square$ & $\square$ & $\square$ & $\square$ & $\square$ & $\square$ & $\square$ & $\begin{array}{l}\text { Uma unidade } \\
\text { média }(75 \mathrm{~g})\end{array}$ & $\square$ & $\square$ & $\square$ & $\square$ \\
\hline Melancia & $\square$ & $\square$ & $\square$ & $\square$ & $\square$ & $\square$ & $\square$ & $\square$ & $\square$ & $\square$ & $\square$ & $\square$ & $\square$ & $\square$ & $\begin{array}{c}\text { Uma fatia } \\
\text { média }(280 \mathrm{~g})\end{array}$ & $\square$ & $\square$ & $\square$ & $\square$ \\
\hline Melão & $\square$ & $\square$ & $\square$ & $\square$ & $\square$ & $\square$ & $\square$ & $\square$ & $\square$ & $\square$ & $\square$ & $\square$ & $\square$ & $\square$ & $\begin{array}{l}\text { Uma fatia } \\
\text { grande } \\
(130 \mathrm{~g})\end{array}$ & $\square$ & $\square$ & $\square$ & $\square$ \\
\hline Tangerina & $\square$ & $\square$ & $\square$ & $\square$ & $\square$ & $\square$ & $\square$ & $\square$ & $\square$ & $\square$ & $\square$ & $\square$ & $\square$ & $\square$ & $\begin{array}{l}\text { Uma unidade } \\
\text { média }(135 \mathrm{~g})\end{array}$ & $\square$ & $\square$ & $\square$ & $\square$ \\
\hline Uva & $\square$ & $\square$ & $\square$ & $\square$ & $\square$ & $\square$ & $\square$ & $\square$ & $\square$ & $\square$ & $\square$ & $\square$ & $\square$ & $\square$ & $\begin{array}{l}\text { Um cacho } \\
\text { pequeno } \\
(170 \mathrm{~g})\end{array}$ & $\square$ & $\square$ & $\square$ & $\square$ \\
\hline
\end{tabular}




\begin{tabular}{|c|c|c|c|c|c|c|c|c|c|c|c|c|c|c|c|c|c|c|c|}
\hline $\begin{array}{l}\text { Carne bovina } \\
\square \text { 1.Cozida } \\
\square \text { 2.Frita } \\
\square 3 \text {. Grelhada/assada }\end{array}$ & $\square$ & $\square$ & $\square$ & $\square$ & $\square$ & $\square$ & $\square$ & $\square$ & $\square$ & $\square$ & $\square$ & $\square$ & $\square$ & $\square$ & $\begin{array}{l}\text { Dois pedaços } \\
\text { grandes ou } \\
\text { um bife } \\
\text { médio }(120 \mathrm{~g})\end{array}$ & $\square$ & $\square$ & $\square$ & $\square$ \\
\hline $\begin{array}{l}\text { Carne de bode } \\
\square \text { 1.Cozida } \\
\square \text { 2.Frita } \\
\square \text { 3.Grelhada/assada }\end{array}$ & $\square$ & $\square$ & $\square$ & $\square$ & $\square$ & $\square$ & $\square$ & $\square$ & $\square$ & $\square$ & $\square$ & $\square$ & $\square$ & $\square$ & $\begin{array}{l}\text { Dois pedaços } \\
\text { grandes } \\
(120 \mathrm{~g})\end{array}$ & $\square$ & $\square$ & $\square$ & $\square$ \\
\hline $\begin{array}{l}\text { Carne de porco } \\
\square \text { 1.Cozida } \\
\square \text { 2.Frita } \\
\square 3 \text {.Grelhada/assada }\end{array}$ & $\square$ & $\square$ & $\square$ & $\square$ & $\square$ & $\square$ & $\square$ & $\square$ & $\square$ & $\square$ & $\square$ & $\square$ & $\square$ & $\square$ & $\begin{array}{l}\text { Dois pedaços } \\
\text { grandes } \\
(120 \mathrm{~g})\end{array}$ & $\square$ & $\square$ & $\square$ & $\square$ \\
\hline $\begin{array}{l}\text { Fígado bovino } \\
\square \text { 1.Cozido } \\
\square \text { 2.Frito } \\
\square 3 \text {.Grelhado/assado }\end{array}$ & $\square$ & $\square$ & $\square$ & $\square$ & $\square$ & $\square$ & $\square$ & $\square$ & $\square$ & $\square$ & $\square$ & $\square$ & $\square$ & $\square$ & $\begin{array}{c}\text { Um bife } \\
\text { médio }(120 \mathrm{~g})\end{array}$ & $\square$ & $\square$ & $\square$ & $\square$ \\
\hline $\begin{array}{l}\text { Frango } \\
\square \text { 1.Cozido } \\
\square \text { 2.Frito } \\
\square 3 \text {.Grelhado/assado }\end{array}$ & $\square$ & $\square$ & $\square$ & $\square$ & $\square$ & $\square$ & $\square$ & $\square$ & $\square$ & $\square$ & $\square$ & $\square$ & $\square$ & $\square$ & $\begin{array}{c}\text { Um pedaço } \\
\text { ou filé médio } \\
(180 \mathrm{~g})\end{array}$ & $\square$ & $\square$ & $\square$ & $\square$ \\
\hline $\begin{array}{l}\text { Peixe } \\
\square \text { 1.Cozido } \\
\square \text { 2.Frito } \\
\square 3 \text {.Grelhado/assado }\end{array}$ & $\square$ & $\square$ & $\square$ & $\square$ & $\square$ & $\square$ & $\square$ & $\square$ & $\square$ & $\square$ & $\square$ & $\square$ & $\square$ & $\square$ & $\begin{array}{l}\text { Uma posta } \\
\text { média }(200 \mathrm{~g})\end{array}$ & $\square$ & $\square$ & $\square$ & $\square$ \\
\hline $\begin{array}{l}\text { Camarão } \\
\square \text { 1.Cozido } \\
\square \text { 2.Frito } \\
\end{array}$ & $\square$ & $\square$ & $\square$ & $\square$ & $\square$ & $\square$ & $\square$ & $\square$ & $\square$ & $\square$ & $\square$ & $\square$ & $\square$ & $\square$ & $\begin{array}{l}\text { Seis unidades } \\
(120 \mathrm{~g})\end{array}$ & $\square$ & $\square$ & $\square$ & $\square$ \\
\hline $\begin{array}{l}\text { Ovo } \\
\square \text { 1.Cozido } \\
\square \text { 2.Frito }\end{array}$ & $\square$ & $\square$ & $\square$ & $\square$ & $\square$ & $\square$ & $\square$ & $\square$ & $\square$ & $\square$ & $\square$ & $\square$ & $\square$ & $\square$ & $\begin{array}{l}\text { Uma unidade } \\
(50 \mathrm{~g})\end{array}$ & $\square$ & $\square$ & $\square$ & $\square$ \\
\hline $\begin{array}{l}\text { Leite de vaca } \\
\text { líquido } \\
\square 1 \text {. Integral } \\
\square 2 \text {. Desnatado }\end{array}$ & $\square$ & $\square$ & $\square$ & $\square$ & $\square$ & $\square$ & $\square$ & $\square$ & $\square$ & $\square$ & $\square$ & $\square$ & $\square$ & $\square$ & $\begin{array}{c}\text { Meia xícara } \\
\text { de chá } \\
(120 \mathrm{~mL})\end{array}$ & $\square$ & $\square$ & $\square$ & $\square$ \\
\hline $\begin{array}{l}\text { Leite de vaca em } \\
\text { pó } \\
\square 1 \text {. Integral } \\
\square 2 . \text { Desnatado }\end{array}$ & $\square$ & $\square$ & $\square$ & $\square$ & $\square$ & $\square$ & $\square$ & $\square$ & $\square$ & $\square$ & $\square$ & $\square$ & $\square$ & $\square$ & $\begin{array}{c}\text { Uma colher } \\
\text { de sopa cheia } \\
(16 \mathrm{~g} / 10 \mathrm{~g})\end{array}$ & $\square$ & $\square$ & $\square$ & $\square$ \\
\hline $\begin{array}{l}\text { Amendoim } \\
\square 1 . \text { Cozido } \\
\square 2 . \text { Torrado }\end{array}$ & $\square$ & $\square$ & $\square$ & $\square$ & $\square$ & $\square$ & $\square$ & $\square$ & $\square$ & $\square$ & $\square$ & $\square$ & $\square$ & $\square$ & $\begin{array}{c}\text { Doze } \\
\text { unidades ou } \\
\text { um punhado } \\
(36 \mathrm{~g}) / \text { Duas } \\
\text { colheres de } \\
\text { sopa cheias } \\
(34 \mathrm{~g})\end{array}$ & $\square$ & $\square$ & $\square$ & $\square$ \\
\hline Feijão preto & $\square$ & $\square$ & $\square$ & $\square$ & $\square$ & $\square$ & $\square$ & $\square$ & $\square$ & $\square$ & $\square$ & $\square$ & $\square$ & $\square$ & $\begin{array}{l}\text { Uma concha } \\
\text { média cheia } \\
(140 \mathrm{~g})\end{array}$ & $\square$ & $\square$ & $\square$ & $\square$ \\
\hline Feijão verde & $\square$ & $\square$ & $\square$ & $\square$ & $\square$ & $\square$ & $\square$ & $\square$ & $\square$ & $\square$ & $\square$ & $\square$ & $\square$ & $\square$ & $\begin{array}{l}\text { Uma concha } \\
\text { média cheia } \\
(140 \mathrm{~g})\end{array}$ & $\square$ & $\square$ & $\square$ & $\square$ \\
\hline $\begin{array}{l}\text { Feijões diversos } \\
\square \text { 1.Carioca ou } \\
\text { mulatinho } \\
\square 2 \text {.Branco } \\
\square 3 \text {. Fradinho }\end{array}$ & $\square$ & $\square$ & $\square$ & $\square$ & $\square$ & $\square$ & $\square$ & $\square$ & $\square$ & $\square$ & $\square$ & $\square$ & $\square$ & $\square$ & $\begin{array}{l}\text { Uma concha } \\
\text { média cheia } \\
\qquad(140 \mathrm{~g})\end{array}$ & $\square$ & $\square$ & $\square$ & $\square$ \\
\hline Castanha de caju & $\square$ & $\square$ & $\square$ & $\square$ & $\square$ & $\square$ & $\square$ & $\square$ & $\square$ & $\square$ & $\square$ & $\square$ & $\square$ & $\square$ & $\begin{array}{l}\text { Oito unidades } \\
\quad(20 \mathrm{~g})\end{array}$ & $\square$ & $\square$ & $\square$ & $\square$ \\
\hline
\end{tabular}




\begin{tabular}{|c|c|c|c|c|c|c|c|c|c|c|c|c|c|c|c|c|c|c|c|}
\hline Castanha do Pará & $\square$ & $\square$ & $\square$ & $\square$ & $\square$ & $\square$ & $\square$ & $\square$ & $\square$ & $\square$ & $\square$ & $\square$ & $\square$ & $\square$ & $\begin{array}{c}\text { Duas } \\
\text { unidades }(8 g)\end{array}$ & $\square$ & $\square$ & $\square$ & $\square$ \\
\hline Cuscuz de milho & $\square$ & $\square$ & $\square$ & $\square$ & $\square$ & $\square$ & $\square$ & $\square$ & $\square$ & $\square$ & $\square$ & $\square$ & $\square$ & $\square$ & $\begin{array}{l}\text { Uma fatia } \\
\text { grande } \\
(135 \mathrm{~g}) \\
\end{array}$ & $\square$ & $\square$ & $\square$ & $\square$ \\
\hline Beiju/Tapioca & $\square$ & $\square$ & $\square$ & $\square$ & $\square$ & $\square$ & $\square$ & $\square$ & $\square$ & $\square$ & $\square$ & $\square$ & $\square$ & $\square$ & $\begin{array}{c}\text { Três colheres } \\
\text { de sopa } \\
\text { cheias }(53 \mathrm{~g})\end{array}$ & $\square$ & $\square$ & $\square$ & $\square$ \\
\hline Aveia em flocos & $\square$ & $\square$ & $\square$ & $\square$ & $\square$ & $\square$ & $\square$ & $\square$ & $\square$ & $\square$ & $\square$ & $\square$ & $\square$ & $\square$ & $\begin{array}{c}\text { Duas } \\
\text { colheres de } \\
\text { sopa cheias } \\
(30 \mathrm{~g}) \\
\end{array}$ & $\square$ & $\square$ & $\square$ & $\square$ \\
\hline $\begin{array}{l}\text { Arroz } \\
\square \text { 1.Branco } \\
\square \text { 2.Integral } \\
\square 3 . \text { Parboilizado }\end{array}$ & $\square$ & $\square$ & $\square$ & $\square$ & $\square$ & $\square$ & $\square$ & $\square$ & $\square$ & $\square$ & $\square$ & $\square$ & $\square$ & $\square$ & $\begin{array}{c}\text { Duas } \\
\text { colheres de } \\
\text { servir cheias } \\
(90 \mathrm{~g})\end{array}$ & $\square$ & $\square$ & $\square$ & $\square$ \\
\hline Milho cozido & $\square$ & $\square$ & $\square$ & $\square$ & $\square$ & $\square$ & $\square$ & $\square$ & $\square$ & $\square$ & $\square$ & $\square$ & $\square$ & $\square$ & $\begin{array}{c}\text { Uma unidade } \\
\text { pequena } \\
(75 \mathrm{~g})\end{array}$ & $\square$ & $\square$ & $\square$ & $\square$ \\
\hline $\begin{array}{l}\text { Farinha de } \\
\text { mandioca }\end{array}$ & $\square$ & $\square$ & $\square$ & $\square$ & $\square$ & $\square$ & $\square$ & $\square$ & $\square$ & $\square$ & $\square$ & $\square$ & $\square$ & $\square$ & $\begin{array}{c}\text { Duas } \\
\text { colheres de } \\
\text { sopa cheias } \\
(22 \mathrm{~g})\end{array}$ & $\square$ & $\square$ & $\square$ & $\square$ \\
\hline Pipoca & $\square$ & $\square$ & $\square$ & $\square$ & $\square$ & $\square$ & $\square$ & $\square$ & $\square$ & $\square$ & $\square$ & $\square$ & $\square$ & $\square$ & $\begin{array}{c}\text { Uma tigela } \\
\text { pequena } \\
\text { cheia }(26 \mathrm{~g})\end{array}$ & $\square$ & $\square$ & $\square$ & $\square$ \\
\hline Café & $\square$ & $\square$ & $\square$ & $\square$ & $\square$ & $\square$ & $\square$ & $\square$ & $\square$ & $\square$ & $\square$ & $\square$ & $\square$ & $\square$ & $\begin{array}{l}\text { Uma xícara } \\
(200 \mathrm{~mL})\end{array}$ & $\square$ & $\square$ & $\square$ & $\square$ \\
\hline $\begin{array}{l}\text { Chá } \\
\text { Sabor: }\end{array}$ & $\square$ & $\square$ & $\square$ & $\square$ & $\square$ & $\square$ & $\square$ & $\square$ & $\square$ & $\square$ & $\square$ & $\square$ & $\square$ & $\square$ & $\begin{array}{l}\text { Uma xícara } \\
(200 \mathrm{~mL})\end{array}$ & $\square$ & $\square$ & $\square$ & $\square$ \\
\hline $\begin{array}{l}\text { Suco de fruta } \\
\text { natural } \\
\text { Sabor: }\end{array}$ & $\square$ & $\square$ & $\square$ & $\square$ & $\square$ & $\square$ & $\square$ & $\square$ & $\square$ & $\square$ & $\square$ & $\square$ & $\square$ & $\square$ & $\begin{array}{l}\text { Um copo } \\
(200 \mathrm{~mL})\end{array}$ & $\square$ & $\square$ & $\square$ & $\square$ \\
\hline
\end{tabular}

\begin{tabular}{|c|c|c|c|c|c|c|c|c|c|c|c|c|c|c|c|c|c|c|c|}
\hline \multirow{2}{*}{$\begin{array}{c}\text { Grupo 2: } \\
\text { Ingredientes } \\
\text { culinários } \\
\end{array}$} & \multicolumn{11}{|c|}{ Quantas vezes você come? } & \multicolumn{3}{|c|}{ Unidade } & Porção média & \multicolumn{4}{|c|}{ Sua porção } \\
\hline & $\mathrm{N}$ & 1 & 2 & 3 & 4 & 5 & 6 & 7 & 8 & 9 & 10 & $\mathrm{D}$ & $\mathrm{S}$ & M & M & $\mathrm{P}$ & M & G & $\mathrm{E}$ \\
\hline Azeite de dendê & $\square$ & $\square$ & $\square$ & $\square$ & $\square$ & $\square$ & $\square$ & $\square$ & $\square$ & $\square$ & $\square$ & $\square$ & $\square$ & $\square$ & $\begin{array}{c}\text { Uma colher } \\
\text { de sobremesa } \\
(5 \mathrm{~mL})\end{array}$ & $\square$ & $\square$ & $\square$ & $\square$ \\
\hline Azeite de oliva & $\square$ & $\square$ & $\square$ & $\square$ & $\square$ & $\square$ & $\square$ & $\square$ & $\square$ & $\square$ & $\square$ & $\square$ & $\square$ & $\square$ & $\begin{array}{c}\text { Uma colher } \\
\text { de sobremesa } \\
(5 \mathrm{~mL})\end{array}$ & $\square$ & $\square$ & $\square$ & $\square$ \\
\hline $\begin{array}{l}\text { Óleo de cozinha } \\
\square \text { 1.Soja } \\
\square 2 \text {.Girassol } \\
\text { Outro: }\end{array}$ & $\square$ & $\square$ & $\square$ & $\square$ & $\square$ & $\square$ & $\square$ & $\square$ & $\square$ & $\square$ & $\square$ & $\square$ & $\square$ & $\square$ & $\begin{array}{c}\text { Uma colher } \\
\text { de sobremesa } \\
(5 \mathrm{~mL})\end{array}$ & $\square$ & $\square$ & $\square$ & $\square$ \\
\hline Manteiga & $\square$ & $\square$ & $\square$ & $\square$ & $\square$ & $\square$ & $\square$ & $\square$ & $\square$ & $\square$ & $\square$ & $\square$ & $\square$ & $\square$ & $\begin{array}{c}\text { Uma colher } \\
\text { de chá cheia } \\
(8 \mathrm{~g})\end{array}$ & $\square$ & $\square$ & $\square$ & $\square$ \\
\hline Margarina & $\square$ & $\square$ & $\square$ & $\square$ & $\square$ & $\square$ & $\square$ & $\square$ & $\square$ & $\square$ & $\square$ & $\square$ & $\square$ & $\square$ & $\begin{array}{c}\text { Uma colher } \\
\text { de chá cheia } \\
(8 g)\end{array}$ & $\square$ & $\square$ & $\square$ & $\square$ \\
\hline $\begin{array}{l}\text { Açúcar } \\
\text { Tipo: }\end{array}$ & $\square$ & $\square$ & $\square$ & $\square$ & $\square$ & $\square$ & $\square$ & $\square$ & $\square$ & $\square$ & $\square$ & $\square$ & $\square$ & $\square$ & $\begin{array}{c}\text { Duas } \\
\text { colheres de } \\
\text { chá cheias } \\
(10 \mathrm{~g})\end{array}$ & $\square$ & $\square$ & $\square$ & $\square$ \\
\hline
\end{tabular}




\begin{tabular}{|l|l|l|l|l|l|l|l|l|l|l|l|l|l|l|l|l|l|l|}
$\square$ & $\square$ & $\square$ & $\square$ & $\square$ & $\square$ & $\square$ & $\square$ & $\square$ & $\square$ & $\square$ & $\square$ & $\square$ & $\square$ & $\begin{array}{c}\text { Uma colher } \\
\text { de chá cheia } \\
(5 \mathrm{~g})\end{array}$ & $\square$ & $\square$ & $\square$ & $\square$ \\
\hline
\end{tabular}

\begin{tabular}{|c|c|c|c|c|c|c|c|c|c|c|c|c|c|c|c|c|c|c|c|}
\hline \multirow{2}{*}{$\begin{array}{c}\text { Grupo 3: } \\
\text { Alimentos } \\
\text { Processados }\end{array}$} & \multicolumn{11}{|c|}{ Quantas vezes você come? } & \multicolumn{3}{|c|}{ Unidade } & Porção média & \multicolumn{4}{|c|}{ Sua porção } \\
\hline & $\mathrm{N}$ & 1 & 2 & 3 & 4 & 5 & 6 & 7 & 8 & 9 & 10 & D & $\mathrm{S}$ & M & M & $P$ & M & G & $\mathrm{E}$ \\
\hline $\begin{array}{l}\text { Queijo } \\
\square \text { 1.Minas } \\
\square \text { 2.Mussarela } \\
\square \text { 3.Ricota } \\
\text { Outro: }\end{array}$ & $\square$ & $\square$ & $\square$ & $\square$ & $\square$ & $\square$ & $\square$ & $\square$ & $\square$ & $\square$ & $\square$ & $\square$ & $\square$ & $\square$ & $\begin{array}{c}\text { Duas fatias } \\
\text { médias } \\
(60 \mathrm{~g} / 40 \mathrm{~g} / 60 \mathrm{~g})\end{array}$ & $\square$ & $\square$ & $\square$ & $\square$ \\
\hline $\begin{array}{l}\text { Carne de } \\
\text { charque/seca/sertão } \\
\square \text { 1.Cozida } \\
\square \text { 2.Frita }\end{array}$ & $\square$ & $\square$ & $\square$ & $\square$ & $\square$ & $\square$ & $\square$ & $\square$ & $\square$ & $\square$ & $\square$ & $\square$ & $\square$ & $\square$ & $\begin{array}{l}\text { Dois pedaços } \\
\text { médios }(60 \mathrm{~g})\end{array}$ & $\square$ & $\square$ & $\square$ & $\square$ \\
\hline Ervilha enlatada & $\square$ & $\square$ & $\square$ & $\square$ & $\square$ & $\square$ & $\square$ & $\square$ & $\square$ & $\square$ & $\square$ & $\square$ & $\square$ & $\square$ & $\begin{array}{c}\text { Uma colher de } \\
\text { sopa cheia } \\
(27 \mathrm{~g})\end{array}$ & $\square$ & $\square$ & $\square$ & $\square$ \\
\hline
\end{tabular}

\begin{tabular}{|c|c|c|c|c|c|c|c|c|c|c|c|c|c|c|c|c|c|c|c|}
\hline \multirow{2}{*}{$\begin{array}{c}\text { Grupo 4: alimentos } \\
\text { ultraprocessados }\end{array}$} & \multicolumn{11}{|c|}{ Quantas vezes você come? } & \multicolumn{3}{|c|}{ Unidade } & Porção média & \multicolumn{4}{|c|}{ Sua porção } \\
\hline & $\mathrm{N}$ & 1 & 2 & 3 & 4 & 5 & 6 & 7 & 8 & 9 & 10 & $\mathrm{D}$ & $S$ & $\mathrm{M}$ & $\mathrm{M}$ & $\mathrm{P}$ & $\mathrm{M}$ & $\mathrm{G}$ & $\mathrm{E}$ \\
\hline $\begin{array}{l}\text { Pão Francês } \\
\square \text { 1.Refinado } \\
\square \text { 2.Integral }\end{array}$ & $\square$ & $\square$ & $\square$ & $\square$ & $\square$ & $\square$ & $\square$ & $\square$ & $\square$ & $\square$ & $\square$ & $\square$ & $\square$ & $\square$ & $\begin{array}{l}\text { Uma unidade } \\
\qquad(50 \mathrm{~g})\end{array}$ & $\square$ & $\square$ & $\square$ & $\square$ \\
\hline $\begin{array}{l}\text { Pães diversos } \\
\square \text { 1.Leite } \\
\square \text { 2.Milho } \\
\square \text { 3.Doce } \\
\end{array}$ & $\square$ & $\square$ & $\square$ & $\square$ & $\square$ & $\square$ & $\square$ & $\square$ & $\square$ & $\square$ & $\square$ & $\square$ & $\square$ & $\square$ & $\begin{array}{l}\text { Uma unidade } \\
(84 \mathrm{~g} / 70 \mathrm{~g} / 77 \mathrm{~g})\end{array}$ & $\square$ & $\square$ & $\square$ & $\square$ \\
\hline $\begin{array}{l}\text { Torrada } \\
\square \text { 1.Refinada } \\
\square \text { 2.Integral }\end{array}$ & $\square$ & $\square$ & $\square$ & $\square$ & $\square$ & $\square$ & $\square$ & $\square$ & $\square$ & $\square$ & $\square$ & $\square$ & $\square$ & $\square$ & $\begin{array}{l}\text { Quatro unidades } \\
(32 \mathrm{~g})\end{array}$ & $\square$ & $\square$ & $\square$ & $\square$ \\
\hline $\begin{array}{l}\text { Bolo simples } \\
\square \text { 1.Trigo } \\
\square \text { 2.Milho } \\
\square \text { 3.Laranja } \\
\text { Outro: }\end{array}$ & $\square$ & $\square$ & $\square$ & $\square$ & $\square$ & $\square$ & $\square$ & $\square$ & $\square$ & $\square$ & $\square$ & $\square$ & $\square$ & $\square$ & $\begin{array}{l}\text { Uma fatia média } \\
(60 \mathrm{~g})\end{array}$ & $\square$ & $\square$ & $\square$ & $\square$ \\
\hline $\begin{array}{l}\text { Biscoito salgado } \\
\square \text { 1.Refinado } \\
\square \text { 2.Integral }\end{array}$ & $\square$ & $\square$ & $\square$ & $\square$ & $\square$ & $\square$ & $\square$ & $\square$ & $\square$ & $\square$ & $\square$ & $\square$ & $\square$ & $\square$ & $\begin{array}{l}\text { Sete unidades } \\
\qquad(43 \mathrm{~g})\end{array}$ & $\square$ & $\square$ & $\square$ & $\square$ \\
\hline $\begin{array}{l}\text { Biscoito doce sem } \\
\text { recheio } \\
\square \text { 1.Refinado } \\
\square \text { 2.Integral }\end{array}$ & $\square$ & $\square$ & $\square$ & $\square$ & $\square$ & $\square$ & $\square$ & $\square$ & $\square$ & $\square$ & $\square$ & $\square$ & $\square$ & $\square$ & $\begin{array}{l}\text { Dez unidades } \\
\quad(55 \mathrm{~g})\end{array}$ & $\square$ & $\square$ & $\square$ & $\square$ \\
\hline $\begin{array}{l}\text { Iogurte } \\
\square 1 \text {. Integral } \\
\square 2 . \text { Desnatado } \\
\text { Sabor: }\end{array}$ & $\square$ & $\square$ & $\square$ & $\square$ & $\square$ & $\square$ & $\square$ & $\square$ & $\square$ & $\square$ & $\square$ & $\square$ & $\square$ & $\square$ & $\begin{array}{l}\text { Um copo } \\
(200 \mathrm{~mL})\end{array}$ & $\square$ & $\square$ & $\square$ & $\square$ \\
\hline $\begin{array}{l}\text { Requeijão } \\
\square 1 \text {. Integral } \\
\square 2 . \text { Light }\end{array}$ & $\square$ & $\square$ & $\square$ & $\square$ & $\square$ & $\square$ & $\square$ & $\square$ & $\square$ & $\square$ & $\square$ & $\square$ & $\square$ & $\square$ & $\begin{array}{c}\text { Uma colher de } \\
\text { sopa cheia }(30 \mathrm{~g})\end{array}$ & $\square$ & $\square$ & $\square$ & $\square$ \\
\hline $\begin{array}{l}\text { Linguiça calabresa } \\
\square \text { 1.Cozida } \\
\square \text { 2.Frita }\end{array}$ & $\square$ & $\square$ & $\square$ & $\square$ & $\square$ & $\square$ & $\square$ & $\square$ & $\square$ & $\square$ & $\square$ & $\square$ & $\square$ & $\square$ & $\begin{array}{l}\text { Dois pedaços } \\
\text { médios }(62 \mathrm{~g})\end{array}$ & $\square$ & $\square$ & $\square$ & $\square$ \\
\hline $\begin{array}{l}\text { Linguiça toscana } \\
\square \text { 1. Assada } \\
\square \text { 2. Frita } \\
\end{array}$ & $\square$ & $\square$ & $\square$ & $\square$ & $\square$ & $\square$ & $\square$ & $\square$ & $\square$ & $\square$ & $\square$ & $\square$ & $\square$ & $\square$ & $\begin{array}{l}\text { Uma unidade } \\
\text { média }(62 \mathrm{~g})\end{array}$ & $\square$ & $\square$ & $\square$ & $\square$ \\
\hline
\end{tabular}


Research, Society and Development, v. 10, n. 16, e280101623506, 2021

(CC BY 4.0) | ISSN 2525-3409 | DOI: http://dx.doi.org/10.33448/rsd-v10i16.23506

\begin{tabular}{|c|c|c|c|c|c|c|c|c|c|c|c|c|c|c|c|c|c|c|c|}
\hline Salsicha & $\square$ & $\square$ & $\square$ & $\square$ & $\square$ & $\square$ & $\square$ & $\square$ & $\square$ & $\square$ & $\square$ & $\square$ & $\square$ & $\square$ & $\begin{array}{l}\text { Duas unidades } \\
\text { médias }(80 \mathrm{~g})\end{array}$ & $\square$ & $\square$ & $\square$ & $\square$ \\
\hline Mortadela & $\square$ & $\square$ & $\square$ & $\square$ & $\square$ & $\square$ & $\square$ & $\square$ & $\square$ & $\square$ & $\square$ & $\square$ & $\square$ & $\square$ & $\begin{array}{l}\text { Duas fatias } \\
\text { médias }(30 \mathrm{~g})\end{array}$ & $\square$ & $\square$ & $\square$ & $\square$ \\
\hline Presunto & $\square$ & $\square$ & $\square$ & $\square$ & $\square$ & $\square$ & $\square$ & $\square$ & $\square$ & $\square$ & $\square$ & $\square$ & $\square$ & $\square$ & $\begin{array}{l}\text { Duas fatias } \\
\text { médias }(30 \mathrm{~g})\end{array}$ & $\square$ & $\square$ & $\square$ & $\square$ \\
\hline $\begin{array}{l}\text { Suco de fruta } \\
\text { industrializado } \\
\text { Sabor/marca: }\end{array}$ & $\square$ & $\square$ & $\square$ & $\square$ & $\square$ & $\square$ & $\square$ & $\square$ & $\square$ & $\square$ & $\square$ & $\square$ & $\square$ & $\square$ & $\begin{array}{l}\text { Um copo } \\
(200 \mathrm{~mL})\end{array}$ & $\square$ & 口 & $\square$ & $\square$ \\
\hline $\begin{array}{l}\text { Refrigerante } \\
\text { Sabor: }\end{array}$ & $\square$ & $\square$ & $\square$ & $\square$ & $\square$ & $\square$ & $\square$ & $\square$ & $\square$ & $\square$ & $\square$ & $\square$ & $\square$ & $\square$ & $\begin{array}{l}\text { Um copo } \\
(200 \mathrm{~mL})\end{array}$ & $\square$ & $\square$ & $\square$ & $\square$ \\
\hline $\begin{array}{l}\text { Pastel } \\
\square \text { 1. Assado } \\
\square \text { 2.Frito } \\
\text { Sabor: }\end{array}$ & $\square$ & $\square$ & $\square$ & $\square$ & $\square$ & $\square$ & $\square$ & $\square$ & $\square$ & $\square$ & $\square$ & $\square$ & $\square$ & $\square$ & $\begin{array}{l}\text { Duas unidades } \\
\text { médias }(120 \mathrm{~g})\end{array}$ & $\square$ & $\square$ & $\square$ & $\square$ \\
\hline $\begin{array}{l}\text { Pizza } \\
\text { Sabor: }\end{array}$ & $\square$ & $\square$ & $\square$ & $\square$ & $\square$ & $\square$ & $\square$ & $\square$ & $\square$ & $\square$ & $\square$ & $\square$ & $\square$ & $\square$ & $\begin{array}{c}\text { Duas fatias } \\
\text { médias }(200 \mathrm{~g})\end{array}$ & $\square$ & $\square$ & $\square$ & $\square$ \\
\hline $\begin{array}{l}\text { Macarrão cozido } \\
\square \text { 1.Refinado } \\
\square \text { 2.Integral }\end{array}$ & $\square$ & $\square$ & $\square$ & $\square$ & $\square$ & $\square$ & $\square$ & $\square$ & $\square$ & $\square$ & $\square$ & $\square$ & $\square$ & $\square$ & $\begin{array}{c}\text { Duas colheres de } \\
\text { servir cheias } \\
(100 \mathrm{~g})\end{array}$ & $\square$ & 口 & $\square$ & $\square$ \\
\hline Achocolatado em pó & $\square$ & $\square$ & $\square$ & $\square$ & $\square$ & $\square$ & $\square$ & $\square$ & $\square$ & $\square$ & $\square$ & $\square$ & $\square$ & $\square$ & $\begin{array}{l}\text { Uma colher de } \\
\text { sopa cheia }(15 \mathrm{~g})\end{array}$ & $\square$ & $\square$ & $\square$ & $\square$ \\
\hline $\begin{array}{l}\text { Chocolate } \\
\text { Sabor: }\end{array}$ & $\square$ & $\square$ & $\square$ & $\square$ & $\square$ & $\square$ & $\square$ & $\square$ & $\square$ & $\square$ & $\square$ & $\square$ & $\square$ & $\square$ & $\begin{array}{c}\text { Dois pedaços } \\
\text { Pequenos }(60 \mathrm{~g})\end{array}$ & $\square$ & $\square$ & $\square$ & $\square$ \\
\hline Goiabada & $\square$ & $\square$ & $\square$ & $\square$ & $\square$ & $\square$ & $\square$ & $\square$ & $\square$ & $\square$ & $\square$ & $\square$ & $\square$ & $\square$ & $\begin{array}{c}\text { Uma fatia média } \\
(66 \mathrm{~g})\end{array}$ & $\square$ & $\square$ & $\square$ & $\square$ \\
\hline $\begin{array}{l}\text { Picolé } \\
\text { Sabor: }\end{array}$ & $\square$ & $\square$ & $\square$ & $\square$ & $\square$ & $\square$ & $\square$ & $\square$ & $\square$ & $\square$ & $\square$ & $\square$ & $\square$ & $\square$ & $\begin{array}{l}\text { Uma unidade } \\
(60 \mathrm{~g})\end{array}$ & $\square$ & $\square$ & $\square$ & $\square$ \\
\hline $\begin{array}{l}\text { Sorvete } \\
\text { Sabor: }\end{array}$ & $\square$ & $\square$ & $\square$ & $\square$ & $\square$ & $\square$ & $\square$ & $\square$ & $\square$ & $\square$ & $\square$ & $\square$ & $\square$ & $\square$ & $\begin{array}{l}\text { Uma bola média } \\
(80 \mathrm{~g})\end{array}$ & $\square$ & $\square$ & $\square$ & $\square$ \\
\hline Extrato de tomate & $\square$ & $\square$ & $\square$ & $\square$ & $\square$ & $\square$ & $\square$ & $\square$ & $\square$ & $\square$ & $\square$ & $\square$ & $\square$ & $\square$ & $\begin{array}{l}\text { Duas colheres de } \\
\text { sopa cheias }(40 \mathrm{~g})\end{array}$ & $\square$ & $\square$ & $\square$ & $\square$ \\
\hline Katchup & $\square$ & $\square$ & $\square$ & $\square$ & $\square$ & $\square$ & $\square$ & $\square$ & $\square$ & $\square$ & $\square$ & $\square$ & $\square$ & $\square$ & $\begin{array}{l}\text { Uma colher de } \\
\text { sopa cheia }(20 \mathrm{~g})\end{array}$ & $\square$ & 口 & $\square$ & $\square$ \\
\hline Maionese & $\square$ & $\square$ & $\square$ & $\square$ & $\square$ & $\square$ & $\square$ & $\square$ & $\square$ & $\square$ & $\square$ & $\square$ & $\square$ & $\square$ & $\begin{array}{c}\text { Uma colher de } \\
\text { sopa cheia }(27 \mathrm{~g})\end{array}$ & $\square$ & 口 & $\square$ & $\square$ \\
\hline
\end{tabular}


Para finalizar, por favor, liste qualquer outro alimento ou preparação importante que você costuma comer ou beber e que não foram citados aqui (por exemplo: leite-de-coco, outros tipos de carnes, receitas caseiras, alguma fruta ou verdura, batata frita, creme de leite, leite condensado, gelatina ou outros doces etc.).

\begin{tabular}{|c|c|c|c|c|c|c|c|c|c|c|c|c|c|c|c|}
\hline \multirow[t]{2}{*}{ Alimento/Preparação } & \multicolumn{11}{|c|}{ Quantas vezes você come? } & \multicolumn{3}{|c|}{ Unidade } & \multirow[t]{2}{*}{ Quantidade consumida } \\
\hline & $\mathrm{N}$ & 1 & 2 & 3 & 4 & 5 & 6 & 7 & 8 & 9 & 10 & $\mathrm{D}$ & $\mathrm{S}$ & $\mathrm{M}$ & \\
\hline & $\square$ & $\square$ & $\square$ & $\square$ & $\square$ & $\square$ & $\square$ & $\square$ & $\square$ & $\square$ & $\square$ & $\square$ & $\square$ & $\square$ & \\
\hline & $\square$ & $\square$ & $\square$ & $\square$ & $\square$ & $\square$ & $\square$ & $\square$ & $\square$ & $\square$ & $\square$ & $\square$ & $\square$ & $\square$ & \\
\hline & $\square$ & $\square$ & $\square$ & $\square$ & $\square$ & $\square$ & $\square$ & $\square$ & $\square$ & $\square$ & $\square$ & $\square$ & $\square$ & $\square$ & \\
\hline & $\square$ & $\square$ & $\square$ & $\square$ & $\square$ & $\square$ & $\square$ & $\square$ & $\square$ & $\square$ & $\square$ & $\square$ & $\square$ & $\square$ & \\
\hline & $\square$ & $\square$ & $\square$ & $\square$ & $\square$ & $\square$ & $\square$ & $\square$ & $\square$ & $\square$ & $\square$ & $\square$ & $\square$ & $\square$ & \\
\hline
\end{tabular}

\section{Outras perguntas:}

Quando você come carne bovina ou suína, costuma comer a gordura visível?
(1) Sim
(2) Não

\section{Quando você come frango ou peixe, costuma comer a pele?}
(1) $\mathrm{Sim}$
(2) Não 
Tabela 4. Lista final dos alimentos presentes no questionário de frequência alimentar, com as respectivas porções em gramas, mililitros e medidas caseiras.

\begin{tabular}{|c|c|c|c|c|}
\hline Grupo de alimentos & $\begin{array}{l}\text { Porção pequena } \\
(\mathrm{p} 25)\end{array}$ & $\begin{array}{l}\text { Porção média } \\
(\mathrm{p} 50)\end{array}$ & Porção grande (p75) & $\begin{array}{l}\text { Porção extragrande } \\
(\mathrm{p} 100)\end{array}$ \\
\hline \multicolumn{5}{|l|}{$\begin{array}{c}\text { Grupo 1: Alimentos in } \\
\text { natura ou } \\
\text { minimamente } \\
\text { processados }\end{array}$} \\
\hline $\begin{array}{l}\text { Abóbora (colher de } \\
\text { sopa cheia) }\end{array}$ & Um (36g) & Duas $(72 \mathrm{~g})$ & Três $(108 \mathrm{~g})$ & Quatro (144g) \\
\hline $\begin{array}{l}\text { Abobrinha (colher de } \\
\text { sopa cheia) }\end{array}$ & $\begin{array}{l}\text { Uma e meia } \\
\qquad(45 \mathrm{~g})\end{array}$ & Três $(90 \mathrm{~g})$ & Cinco $(150 g)$ & Seis $(180 \mathrm{~g})$ \\
\hline $\begin{array}{l}\text { Acelga (colher de sopa } \\
\text { cheia) }\end{array}$ & Duas (12g) & Três (18g) & Quatro (24g) & Seis $(36 \mathrm{~g})$ \\
\hline Alface (folhas grandes) & Uma (15g) & Duas $(30 \mathrm{~g})$ & Três (45g) & Seis $(90 \mathrm{~g})$ \\
\hline $\begin{array}{l}\text { Rúcula (colher de sopa } \\
\text { cheia) }\end{array}$ & Duas $(16 \mathrm{~g})$ & Quatro (32g) & Seis $(48 g)$ & Oito $(64 g)$ \\
\hline Couve (folha média) & Uma $(20 \mathrm{~g})$ & Duas (40g) & Três $(60 \mathrm{~g})$ & Quatro (80g) \\
\hline $\begin{array}{l}\text { Repolho (colher de } \\
\text { sopa cheia) }\end{array}$ & Duas $(20 g)$ & Três $(30 \mathrm{~g})$ & Quatro (40g) & Seis $(60 \mathrm{~g})$ \\
\hline $\begin{array}{l}\text { Couve-flor (ramos } \\
\text { pequenos) }\end{array}$ & Dois $(60 \mathrm{~g})$ & Três $(90 \mathrm{~g})$ & Quatro (120g) & Seis $(180 \mathrm{~g})$ \\
\hline $\begin{array}{l}\text { Cebola (colher de sopa } \\
\text { cheia) }\end{array}$ & Uma (10g) & Duas $(20 \mathrm{~g})$ & Três $(30 \mathrm{~g})$ & Quatro (40g) \\
\hline $\begin{array}{l}\text { Chuchu (colher de sopa } \\
\text { cheia) }\end{array}$ & Uma (20g) & Duas $(40 g)$ & Três $(60 \mathrm{~g})$ & Seis $(120 g)$ \\
\hline $\begin{array}{l}\text { Pepino (colher de sopa } \\
\text { cheia) }\end{array}$ & Uma (13g) & Duas $(26 g)$ & Três (39g) & Seis $(78 \mathrm{~g})$ \\
\hline Jiló (unidade) & $\begin{array}{l}\text { Uma pequena } \\
(27 \mathrm{~g})\end{array}$ & Uma média (39g) & Uma grande $(63 g)$ & Duas médias (78g) \\
\hline Tomate (unidade) & $\begin{array}{l}1 / 2 \text { pequena } \\
(30 \mathrm{~g})\end{array}$ & $\begin{array}{l}\text { Uma pequena } \\
\qquad(60 \mathrm{~g})\end{array}$ & Uma média (100g) & Uma grande (150g) \\
\hline Maxixe (unidade) & Uma média (13g) & $\begin{array}{l}\text { Duas grandes } \\
\qquad(40 \mathrm{~g})\end{array}$ & $\begin{array}{l}\text { Quatro grandes } \\
\qquad(80 \mathrm{~g})\end{array}$ & Cinco grandes $(100 \mathrm{~g})$ \\
\hline Quiabo (unidade) & $\begin{array}{l}\text { Duas médias } \\
\qquad(23 \mathrm{~g})\end{array}$ & $\begin{array}{l}\text { Duas grandes } \\
\qquad(40 \mathrm{~g})\end{array}$ & Três grandes $(60 \mathrm{~g})$ & Quatro grandes $(80 \mathrm{~g})$ \\
\hline $\begin{array}{l}\text { Vagem (colher de sopa } \\
\text { cheia) }\end{array}$ & Duas $(40 \mathrm{~g})$ & Quatro (80g) & Seis $(120 g)$ & Oito $(180 \mathrm{~g})$ \\
\hline $\begin{array}{l}\text { Beterraba (colher de } \\
\text { sopa cheia) }\end{array}$ & Uma (20g) & Duas $(40 \mathrm{~g})$ & Seis $(60 g)$ & Oito $(80 \mathrm{~g})$ \\
\hline $\begin{array}{l}\text { Cenoura (colher de } \\
\text { sopa cheia) }\end{array}$ & Uma (25g) & Duas $(50 \mathrm{~g})$ & Três $(75 \mathrm{~g})$ & Seis $(150 g)$ \\
\hline $\begin{array}{c}\text { Banana da terra cozida } \\
\text { (Unidade) }\end{array}$ & 1/5 média $(60 \mathrm{~g})$ & $\begin{array}{l}\text { Uma pequena } \\
\qquad(107 \mathrm{~g})\end{array}$ & Uma média (112g) & Uma grande $(221 \mathrm{~g})$ \\
\hline $\begin{array}{l}\text { Inhame cozido (fatia } \\
\text { média) }\end{array}$ & Uma (86g) & Duas $(172 \mathrm{~g})$ & Três $(258 \mathrm{~g})$ & Quatro (344g) \\
\hline $\begin{array}{l}\text { Aipim cozido (pedaço } \\
\text { médio) }\end{array}$ & Um $(100 \mathrm{~g})$ & Dois $(200 \mathrm{~g})$ & Três $(300 \mathrm{~g})$ & Quatro (400g) \\
\hline $\begin{array}{l}\text { Batata doce cozida } \\
\text { (pedaço médio) }\end{array}$ & $\operatorname{Um}(70 \mathrm{~g})$ & Dois $(140 \mathrm{~g})$ & Dois $(210 \mathrm{~g})$ & Três $(280 \mathrm{~g})$ \\
\hline
\end{tabular}


Batata inglesa cozida (colher de sopa cheia)

Abacaxi (rodela média)

Banana prata (unidade)

Goiaba (unidade)

Maçã (unidade)

Laranja (unidade)

Mamão (fatia)

Manga (unidade)

Melancia (fatia)

Melão (fatia)

Tangerina (unidade)

Uva (cacho)

Carne bovina (pedaço)

Carne de bode (pedaço)

Carne de porco (pedaço)

Fígado bovino (bife ou fatia)

Frango (pedaço ou filé)

Peixe (posta)

Camarão (unidade grande)

Ovo (unidade)

Leite de vaca líquido (xícara de chá)

Leite de vaca em pó integral (colher de sopa)

Leite de vaca em pó desnatado (colher de sopa)

Amendoim cozido (unidades)

Amendoim torrado (colher de sopa cheia)

Feijão preto (concha média)

Feijão verde (concha média)

\section{Uma (30g)}

Uma (89g)

Uma pequena (41g)

Uma pequena (100g)

Uma pequena (100g)

Uma pequena (88g)

Uma pequena (43g)

Uma pequena (54g)

Uma pequena (200g)

Uma média (87g)

Uma pequena (100g)

$1 / 2$ pequeno $(85 \mathrm{~g})$

Três médios (90g)

Três médios (90g)

Três médios (90g)

Um pequeno (80g)

Um pequeno $(140 \mathrm{~g})$

Uma pequena (150g)

Três $(60 \mathrm{~g})$

Meia (25g)

Um terço $(80 \mathrm{~mL})$

Uma rasa (8g) Uma cheia (16g)

Uma rasa $(8 g)$

Uma cheia (10g)

Seis $(18 g)$

Uma (17g)

Uma rasa (80g)

Doze ou um punhado (36g)

Duas (34g)

Uma cheia $(140 \mathrm{~g})$

Uma rasa $(80 g)$
Duas cheias (32g)

Duas cheias (280g)

Quatro (120g)

Oito $(240 \mathrm{~g})$

Três (267g)

Quatro (356g)

Três médias (180g)

Duas médias (340g)

Duas médias (260g)

Duas médias (340g)

Duas grandes (276g)

Duas grandes (230g)

Duas médias (560g)

Duas grandes (260g)

Duas grandes (540g)

Dois pequenos $(340 \mathrm{~g})$

Um grande (560g)

Três grandes (180g) Cinco grandes (300g)

Três grandes (180g) Cinco grandes $(300 \mathrm{~g})$

Três grandes (180g) Cinco grandes (300g)

Dois médios (240g) Dois grandes (300g)

Um grande (270g) Dois médios (360g)

Uma grande (250g) Duas médias (400g)

Nove (180g)

Doze (240g)

Duas (100g)

Uma cheia

(240mL)

Três unidades $(150 \mathrm{~g})$

Duas cheias $(480 \mathrm{~mL})$

Quatro cheias (64g)

Quatro cheias (40g)

Duas cheias (20g)

Dezoito (54g)

Três $(51 \mathrm{~g})$

Quatro (68g)

Três cheias (420g)

Três cheias (420g) 
Feijões diversos (concha média)

Castanha de caju (unidade)

Castanha do Pará (unidade)

Cuscuz de milho (fatia)

Tapioca (colher de sopa cheia)

Aveia em flocos (colher de sopa cheia)

Arroz (colher de servir cheia)

Milho cozido (unidade)

Farinha de mandioca (colher de sopa cheia)

Pipoca (uma tigela pequena)

Café (xícara/caneca)

Chá (xícara/caneca)

Suco de fruta natural (copo)

\section{Grupo 2: Ingredientes} culinários

Azeite de dendê (colher)

Azeite de oliva (colher)

Óleo de cozinha (colher)

Manteiga (colher de chá)

Margarina

Açúcar (colher de chá)

Sal (colher de chá)

Grupo 3: Alimentos Processados

Queijo minas (fatia média)

Uma (30g)

Duas (60g)

Duas (40g)

$$
\begin{aligned}
& \text { Três }(7,5 g) \\
& \text { Uma }(4 g)
\end{aligned}
$$

Uma média $(79 \mathrm{~g})$

Duas (35g)

Uma (15g)

Uma (45g)

$1 / 2$ pequena $(37 \mathrm{~g})$

Uma (11g)

Rasa (15)

Uma pequena (150mL)

Uma pequena (150mL)

$1 / 2$ médio

$(100 \mathrm{~mL})$

Uma de chá

( $2 \mathrm{~mL})$

Uma de chá ( $2 \mathrm{~mL})$

Uma de chá

(2mL)

Uma rasa (4g)

Uma rasa $(4 g)$

Uma cheia (5g)

Uma rasa $(3,7 \mathrm{~g})$
Oito (20g)

Duas $(8 \mathrm{~g})$

Uma grande (135g)

Três $(53 g)$

Duas (30g)

Duas (90g)

Uma pequena (75g)

Duas (22)

Cheia (26g)

Uma (200mL)

Uma (200mL)

Um médio

(200mL)

Uma de sobremesa $(5 \mathrm{~mL})$

Uma de sobremesa $(5 \mathrm{~mL})$

Uma de sobremesa $(5 \mathrm{~mL})$

Uma cheia (8g)

Uma cheia $(8 g)$

Duas cheias (10g)

Uma cheia $(5 g)$
Queijo mussarela (fatia média)

Uma (20g)

Duas cheias $(280 \mathrm{~g})$

Duas cheias (420g)

$12(30 \mathrm{~g})$

Três $(12 \mathrm{~g})$

Duas médias (158g)

Sete (123g)

Três $(45 \mathrm{~g})$

Três (135g)

Sete (315g)

Uma média (92g)

Uma grande (129g)

Três (33g)

Oito $(89 g)$

Duas cheias (52g)

Uma média (300mL)

Uma média (300mL)

Um grande $(300 \mathrm{~mL})$

Uma de sopa (8mL)

Uma de sopa $(8 \mathrm{~mL})$

Uma de sopa (8mL)

Duas cheias (16g)

Duas cheias (16g)

Três cheias (15g)

Duas cheias (10g)
Duas de sobremesa (10mL)

Duas de sobremesa $(10 \mathrm{~mL})$

Duas de sobremesa $(10 \mathrm{~mL})$

Três cheias (24g)

Três cheias $(24 \mathrm{~g})$

Cinco cheias (25g)

Três cheias $(15 \mathrm{~g})$

Três $(60 \mathrm{~g})$

Quatro (80g) 
Queijo ricota (fatia média)

Uma $(30 g)$

$\operatorname{Um}(30 \mathrm{~g})$

Dois $(60 \mathrm{~g})$

Carne de charque

(pedaço médio)

Ervilha enlatada (colher de sopa)

\section{Grupo 4: alimentos ultraprocessados}

Pão Francês (unidade)

Pães de leite (unidade)

Pão de milho (unidade)

Pão doce (unidade)

Torrada (unidade)

Bolo simples (fatia)

Biscoito salgado (unidade)

Biscoito doce (unidade)

Iogurte (copo)

Requeijão (colher de sopa)

Linguiça calabresa

(pedaço médio)

\section{Linguiça toscana} (unidade)

Salsicha (unidade média)

Mortadela (fatia média)

Presunto (fatia média)

Suco de fruta industrializado (copo)

Refrigerante (copo)

Pastel (unidade)

$$
\text { Pizza (fatia) }
$$

Macarrão cozido (colher de servir)

Achocolatado em pó (colher de sopa)

Chocolate (pedaço pequeno)

Uma rasa (16g)

Uma (50g)

Meia (42g)

Uma (84g)

Meia (35g)

Meia $(38,5 \mathrm{~g})$

Duas (16g)

Pequena (30g)

Uma (77g)

Quatro (32g)

Média (60g)

Sete $(43 g)$

$\operatorname{Dez}(55 \mathrm{~g})$

Meio (100mL)

Uma rasa (15g)

$\mathrm{Um}(31 \mathrm{~g})$

Uma pequena (47g)

$$
\begin{aligned}
& \text { Uma (40g) } \\
& \text { Uma (15g) }
\end{aligned}
$$

Uma (15g)

$1 / 2$ médio

(100mL)

$1 / 2$ médio

(100mL)

Uma grande (80g)

Uma grande (130g)

Uma cheia $(50 \mathrm{~g})$

Dois (62g)

Duas (80g)

Duas (30g)

Duas (30g)

Um médio (200mL)

Um médio (200mL) (120g) (260g)

Duas cheias (100g)

Uma (30g)
Uma cheia (27g)

Duas (100g)

Três (150g)

Duas (168g)

Três (252g)

\section{Uma (70g)}

Um (200mL)

Uma cheia (30g)

Uma média (62g)

Duas médias

Duas grandes

Uma rasa (10g) Uma cheia (15g)
Uma e meia (105g)

Uma e meia $(115 \mathrm{~g})$

Sete $(56 \mathrm{~g})$

Grande (100g)

Doze (73)

Quinze (82)

Um e meio

(300mL)

1,5 cheia $(45 \mathrm{~g})$

Quatro (124g)

Duas médias (124g)

Três (120g)

Três $(45 \mathrm{~g})$

Três $(45 \mathrm{~g})$

Um grande $(300 \mathrm{~mL})$

Um grande $(300 \mathrm{~mL})$

Três médias $(180 \mathrm{~g})$

Quatro grandes (520g)

Três cheias (150g)

Duas rasas (20g)

Duas cheias (30g)

Duas (140g)

Duas (154g)

Dez (80g)

Duas médias (120g)

Vinte (122)

Vinte (110g)

Dois $(400 \mathrm{~mL})$

Duas cheias $(60 \mathrm{~g})$

Seis (186g)

Três médias (186g)

Quatro (180g)

Quatro (60g)

Quatro (60g)

Dois médios (400mL)

Dois médios (400mL)

Três grandes (240g)

Sete grandes (910g)

Quatro cheias (200g)

Três $(90 \mathrm{~g})$
Quatro (120g) 


$\begin{array}{ccccc}\text { Goiabada (fatia) } & \text { Uma pequena } & \text { Uma média }(66 \mathrm{~g}) & \begin{array}{c}\text { Duas pequenas } \\ (96 \mathrm{~g})\end{array} & \text { Duas médias }(132 \mathrm{~g}) \\ & (48 \mathrm{~g}) & \text { Uma }(60 \mathrm{~g}) & \text { Duas }(120 \mathrm{~g}) & \text { Três }(180 \mathrm{~g}) \\ \text { Picolé (unidade) } & 1 / 2(30 \mathrm{~g}) & \text { Uma }(80 \mathrm{~g}) & \text { Duas }(160 \mathrm{~g}) & \text { Três }(240 \mathrm{~g})\end{array}$

Extrato de tomate (colher de sopa)

Katchup (colher de sopa)

Maionese (colher de sopa)
Uma cheia (20g) Duas cheias (40g) Três cheias (60g) Quatro cheias (80g)

Uma rasa $(11 \mathrm{~g}) \quad$ Uma cheia $(20 \mathrm{~g}) \quad$ Duas cheias $(40 \mathrm{~g}) \quad$ Três cheias $(60 \mathrm{~g})$

Uma rasa (17g) Uma cheia $(27 \mathrm{~g}) \quad$ Duas rasas $(34 \mathrm{~g}) \quad$ Duas cheias $(54 \mathrm{~g})$

Fonte: Autores (2021).

\section{Discussão}

O presente estudo não identificou diferença no consumo total de energia, macronutrientes e micronutrientes entre os pacientes com esteatose leve ou moderada. Todavia, algumas variações significativas foram observadas na ingestão alimentar segundo o grau de processamento dos alimentos. O consumo de alimentos processados e ultraprocessados foram citados com frequência por ambos os grupos, mas no geral a ingestão de alimentos in natura ou minimamente processados se mostrou mais frequente na população avaliada, similar ao padrão alimentar da população Brasileira (Instituto Brasileiro de Geografia e Estatística [IBGE] 2011; IBGE, 2020).

Maior ingestão de alimentos in natura ou minimamente processados é um resultado favorável para este grupo, pois o consumo de alimentos processados e ultraprocessados pode favorecer o ganho de peso pela presença de grandes quantidades de calorias, açúcar, gorduras saturadas, gorduras trans e elevada densidade energética, além do baixo teor de micronutrientes e, consequentemente, associar-se ao avanço da esteatose hepática (Poti et al., 2017; Rico-Campa et al., 2019; Hall et al., 2019).

Dentre os pacientes avaliados, aqueles com esteatose moderada apresentaram maiores valores de CC, achados que corroboram com outros dados da literatura, os quais associam a DHGNA à outras doenças metabólicas, como obesidade, especialmente na região abdominal, diabetes mellitus tipo 2 e dislipidemia (Lu et al., 2018; Younossi et al., 2019). Entretanto, apesar da associação amplamente descrita sobre a correlação direta entre consumo alimentar e obesidade, neste trabalho não foi verificada correlação significativa entre os valores de CC ou IMC com a ingestão dos grupos alimentares avaliados.

Diante desses resultados, não podemos descartar a possibilidade de subestimação das informações relatadas sobre o consumo alimentar, a partir dos R24h, tendo em vista que os baixos valores calóricos observados em ambos os grupos não são comuns em pacientes com DHGNA, especialmente quando não recebem orientação nutricional prévia. O estudo de Crispim et al. (2016), realizado com 158 pacientes com DHGNA de um ambulatório público da cidade de São Paulo, observou que o consumo alimentar foi superior em aproximadamente $500 \mathrm{Kcal}$ para os pacientes sem orientação nutricional prévia, quando comparado ao grupo com orientação nutricional anterior.

Um possível sub-relato no consumo alimentar por estes pacientes pode ter implicado também na avaliação do consumo de micronutrientes e macronutrientes e na observação das associações investigadas. A avaliação da mediana do consumo calórico por quilo de peso dos pacientes apontou valores inferiores a $20 \mathrm{kcal} / \mathrm{kg}$, sugerido apenas para pacientes obesos com acompanhamento especializado (McClave et al., 2016; Bischoff et al., 2020). Estes achados corroboram com relatos na literatura quanto a subestimação do consumo alimentar entre indivíduos com excesso de peso, achados denominado de causalidade reversa (Avelino et al., 2014; Associação Brasileira para o Estudo da Obesidade [ABESO], 2016). 
Registra-se ainda limitações na quantificação do consumo alimentar relacionadas as informações nutricionais incompletas nos rótulos de diversos alimentos processados e ultraprocessados e a ausência dessas informações em tabelas de composição de alimentos, o que pode ter promovido uma quantificação menor de energia e nutrientes.

No presente estudo os pacientes com esteatose leve apresentaram um maior consumo de carboidratos e lipídios totais derivados de ingredientes culinários, especialmente açúcar refinado, óleo e margarina alimentos cuja ingestão elevada pode favorecer a evolução DHGNA (Jensen et al., 2018). No entanto, esses pacientes também apresentaram um maior consumo de lipídios poli-insaturados provenientes de alimentos in natura ou minimamente processados, resultado que pode ser analisado como um ponto positivo e de possível equilíbrio na ingestão alimentar, pois o aumento do consumo desse tipo de lipídio relaciona-se a redução da resistência à insulina e consequentemente melhora da DHGNA (Sofi et al., 2010; Kirpich et al., 2016).

As principais fontes de cálcio foram os alimentos in natura ou minimamente processados, com grande contribuição do leite, alimento lácteo mais citado neste grupo. Porém, o consumo desse nutriente a partir de alimentos in natura ou minimamente processados não apresenta diferença significante segundo o grau de esteatose. Por outro lado, o cálcio proveniente dos alimentos processados ou ultraprocessados foi significativamente maior entre os indivíduos com esteatose leve. De modo geral, as principais fontes de cálcio presentes entre os alimentos processados e ultraprocessados são os derivados do leite, como queijo, iogurte e requeijão, mas entre os pacientes avaliados apenas o queijo foi citado com maior frequência.

Por outro lado, a ingestão de vitamina A, proveniente de fontes in natura ou minimamente processadas, foi maior entre os indivíduos com esteatose moderada. O maior consumo de alimentos deste grupo é o mais recomendado para o tratamento da DHGNA (Chalasani et al., 2018; Arab et al., 2020). Entre esses alimentos o café teve o maior percentual de citação, com o consumo médio de $200 \mathrm{ml}$ e máximo de $400 \mathrm{ml}$. Esse dado pode ser um ponto positivo, pois estudos atuais recomendam consumo moderado, definidas como duas ou três xícaras de café por dia, para os pacientes com DHGNA devido ao efeito antioxidante dessa bebida (Arab et al., 2020; Bambha et al., 2014).

A classificação dos alimentos com base na extensão do processamento industrial realizada neste estudo demonstrou que os alimentos mais consumidos foram aqueles tipicamente presentes na dieta básica da população brasileira, de acordo com as duas últimas Pesquisas de Orçamento Familiar (POF) realizadas no país: café, feijão, arroz, frango, leite, banana, açúcar, óleo de soja, margarina, pão francês, biscoitos salgados, macarrão, carne de charque e queijos (IBGE, 2011; IBGE, 2020).

Dados do mais recente inquérito nacional sobre alimentação, realizado com 46.164 indivíduos com idade $\geq 10$ anos incluídos na POF de 2017-2018, revelaram que 53,4\% das calorias consumidas foram provenientes de alimentos in natura ou minimamente processados, $15,6 \%$ de ingredientes culinários, $11,3 \%$ de alimentos processados e 19,7\% de alimentos ultraprocessados (IBGE, 2020). Ressalta-se que o consumo total de alimentos processados e ultraprocessados identificados pela POF apresenta valor semelhante ao percentual que essa categoria ocupou entre os alimentos presentes no QFA proposto (31\% vs. $30,2 \%)$.

Diversos QFAs foram desenvolvidos e validados para a população brasileira, mas com a finalidade de avaliar a ingestão alimentar de indivíduos com DHGNA identificamos apenas uma publicação desenvolvida na Irlanda que apresentou e validou um QFA semiquantitativo, elaborado a partir de dados de instrumentos previamente validados para outros grupos populacionais e posteriormente adaptado para indivíduos com DHGNA (Bredin et al., 2020).

Destacamos a relevância do desenvolvimento de um QFA a partir de informações alimentares de indivíduos com DHGNA visto que, para avaliar a ingestão alimentar de forma mais fidedigna, este instrumento deve ser desenvolvido e validado para a população de interesse. O QFA aqui proposto atende também as questões metodológicas no que se refere ao tamanho da lista de alimentos, a qual deve variar entre 50 e 100 itens (Block et al., 1985; Slater et al., 2003; Cade et al., 2002; Cade et al., 2004). 
O período de referência do QFA pode variar entre o consumo do ano precedente ou até do mês anterior ao estudo (Cade et al., 2002; Cade et al., 2004). Nesta publicação o período mínimo de recordação foi definido como o mês anterior, no entanto o prazo de avaliação pode ser adaptado em estudos posteriores que avaliem a validade e reprodutibilidade do questionário, passo fundamental para garantir informações mais precisas sobre o consumo alimentar dessa população.

Destacamos como pontos fortes deste estudo o rigor metodológico adotado desde a coleta, codificação e análise dos dados até o processo de seleção e construção da lista de alimentos para o desenvolvimento do QFA aqui proposto. Uma limitação foi a impossibilidade de realizar a validação do QFA desenvolvido. A etapa de validação precisou ser interrompida devido a suspensão dos atendimentos presenciais diante da atual pandemia da COVID-19 provocada pelo novo coronavírus (SARS-CoV2: severe acute respiratory syndrome coronavirus 2) (Wang et al., 2020; WHO, 2021). Assim, a medição da reprodutibilidade e validade do instrumento será realizada em pesquisas futuras.

\section{Conclusão}

Os pacientes com DHGNA apresentaram consumo alimentar similar ao padrão básico da população brasileira e, apesar da ingestão elevada de ultraprocessados, os alimentos in natura ou minimamente processados foram predominantes. A análise da ingestão alimentar habitual, considerando o grau de processamento, mostrou diferença significante para o consumo de alguns nutrientes entre os indivíduos com esteatose leve e moderada. No entanto, o consumo total de macro e micronutrientes destes pacientes não se correlacionou com a gravidade da esteatose, nem com os dados antropométricos. Mas, os pacientes com esteatose moderada apresentaram valores mais elevados da circunferência da cintura.

O QFA quantitativo desenvolvido neste estudo caracteriza-se como um instrumento prático para avaliação do consumo alimentar habitual de pacientes com DHGNA e apresenta alimentos que fazem parte da alimentação brasileira habitual. Considerando a participação importante da alimentação no controle metabólico da DHGNA, espera-se que o instrumento desenvolvido seja útil para avaliar a ingestão de nutrientes e monitorar as práticas alimentares desta população, e que possa ser utilizado em estudos posteriores para investigar associações entre dieta e a presença ou evolução da esteatose hepática.

\section{Agradecimentos}

Os autores agradecem à Fundação de Amparo à Pesquisa da Bahia (FAPESB) pelo financiamento da bolsa de doutorado da autora Laís Spíndola Garcêz.

\section{Referências}

Arab, J. P., Dirchwolf, M., Álvares-da-Silva M. R., Barrera, F., Benítez, C., Castellanos-Fernandez, M. \& Arrese, M. (2020). Latin American Association for the study of the liver (ALEH) practice guidance for the diagnosis and treatment of non-alcoholic fatty liver disease. Annals of Hepatology, 19(6), 674-690. 10.1016/j.aohep.2020.09.006

Associação Brasileira para o Estudo da Obesidade e da Síndrome Metabólica (ABESO). (2016). Diretrizes brasileiras de obesidade. 4ed. São Paulo: ABESO. Disponível em: https://abeso.org.br/wp-content/uploads/2019/12/Diretrizes-Download-Diretrizes-Brasileiras-de-Obesidade-2016.pdf

Avelino, G.F., Previdelli, N. A., Castro, M. A., Marchioni, D. M. L., \& Fisberg, R. M. (2014). Sub-relato da ingestão energética e fatores associados em estudo de base populacional. Cadernos de Saúde Pública, 30(3), 663-668. http://dx.doi.org/10.1590/0102-311X00073713

Bambha, K., Wilson, L. A., Unalp, A., Loomba, R., Neuschwander-Tetri, B.A., Brunt, E. M., \& Bass N. M. (2014). Coffee consumption in NAFLD patients with lower insulin resistance is associated with lower risk of severe fibrosis. Liver International, 34(8), 1250-1258. 10.1111/liv.12379

Berquó, E. (1981). Bioestatística. E.P.U.

Bischoff, S. C., Bernal, W., Dasarathy, S., Merli, M., Plank, L. D., Schütz, T., \& Plauth, M. (2020). ESPEN practical guideline: Clinical nutrition in liver disease. Clinical Nutrition, 39(12), 3533-3562. 10.1016/j.clnu.2020.09.001

Block, G., Dresser, C. M., Hartman, A. M., \& Carroll, M. D. (1985). Nutrient sources in the American diet: quantitative data from the NHANES II survey. II. Macronutrients and fats. The American Journal of Epidemiology, 122(1), 27-40. 10.1093/oxfordjournals.aje.a114084 
Block, G., Hartman, A. M., Dresser, C. M., Carroll, M. D., Gannon, J., \& Gardner, L. (1986). A data-based approach to diet questionnaire design and testing. The American Journal of Epidemiology, 124(3), 453-469. 10.1093/oxfordjournals.aje.a114416

Brasil. Ministério da Saúde. Instituto Nacional de Alimentação e Nutrição. (1996). Registro fotográfico para inquéritos dietéticos - utensílios e porções. Ministério da Saúde.

Brasil. Ministério da Saúde. Secretaria de Atenção à Saúde. (2014). Guia alimentar para a população brasileira. 2.ed. Brasília: Ministério da Saúde. https://www.gov.br/saude/pt-br/assuntos/saude-brasil/publicacoes-para-promocao-a-saude/guia_alimentar_populacao_brasileira_2ed.pdf/view

Bredin, C., Naimimohasses, S. Norris, S., Wright, C., Hancock, N., \& Hart, K., Moore, J. B. (2020). Development and relative validation of a short food frequency questionnaire for assessing dietary intakes of non-alcoholic fatty liver disease patients. European Journal of Nutrition, 59(2), 571-580. https://doi.org/10.1007/s00394-019-01926-5

Buzzard, M. (1998). 24-hours dietary recall and food record methods. In: Willett WC. Nutritional Epidemiology. 2ed. Oxford: Oxford University Press.

Cade, J., Thompson, R., Burley, V., \& Warm, D. (2002). Development, validation and utilisation of food-frequency questionnaires - a review. Public Health Nutrition, 5(4), 567-587. 10.1079/PHN2001318

Cade, J. E., Burley, V. J., Warm, D. L., Thompson, R. L., \& Margetts, B. M. (2004). Food-frequency questionnaires: a review of their design, validation and utilisation. Nutrition Research Reviews, 17(1), 5-22. 10.1079/NRR200370

Cardoso, M. A., \& Stocco, P. R. (2000). Desenvolvimento de um questionário quantitativo de frequência alimentar em imigrantes japoneses e seus descendentes residentes em São Paulo, Brasil. Cadernos de Saúde Pública, 16(1), 107-114. https://doi.org/10.1590/S0102-311X2000000100011

Chalasani, N., Younossi, Z., Lavine, J. E., Charlton, M., Cusi, K., Rinella, M., Harrison, S.A., Brunt, E. M., \& Arun, J. S. (2018). The diagnosis and management of nonalcoholic fatty liver disease: practice guidance from the American Association for the Study of Liver Diseases. Hepatology, 67(1), 328-357. 10.1002/hep.29367

Chen, X., Zhang, Z., Yang, H., Qiu, P., Wang, H., Wang, F., Zhao, Q., Fang, J., \& Nie, J. (2020). Consumption of ultra-processed foods and health outcomes: a systematic review of epidemiological studies. Nutrition journal, 19(1). 10.1186/s12937-020-00604-1

Crispim, F. G. S., Elias, M. C., \& Parise, E. R. (2016). Consumo alimentar dos portadores de Doença Hepática Gordurosa Não Alcoólica: comparação entre a presença e a ausência de Esteatoepatite Não Alcoólica e Síndrome Metabólica. Revista de Nutrição, 29(4), 495-505. https://doi.org/10.1590/167898652016000400005

Eslam, M., Sanyal, A. J., \& George, J. (2020). International Consensus Panel. MAFLD: A Consensus-Driven Proposed Nomenclature for Metabolic Associated Fatty Liver Disease. Gastroenterology, 158(7), 1999-2014. 10.1053/j.gastro.2019.11.312

Estes, C., Razavi, H., Loomba, R., Younossi, Z., \& Sanyal, A.J. (2018). Modeling the epidemic of nonalcoholic fatty liver disease demonstrates an exponential increase in burden of disease. Hepatology, 67(1), 23-133. 10.1002/hep.29466

Fisberg, R. M., Colucci, A. C., Morimoto, J. M., \& Marchioni, D. M. (2008). Questionário de frequência alimentar para adultos com base em estudo populacional Revista de Saúde Pública, 42(3), 550-554. https://doi.org/10.1590/S0034-89102008005000020

Fisberg, R. M., Marchioni, D. M., \& Colucci, A. C. (2009). Avaliação do consumo alimentar e da ingestão de nutrientes na prática clínica. Arquivos Brasileiros de Endocrinologia \& Metabologia, 53(5), 617-624. https://doi.org/10.1590/S0004-27302009000500014

Garcêz, L. S., Avelar, C. R., Fonseca, N. S. S., Costa P. R. F., Lyra A. C., Cunha C. M., Jesus R. P., \& Oliveira, L. P. M. (2021). Effect of dietary carbohydrate and lipid modification on clinical and anthropometric parameters in nonalcoholic fatty liver disease: a systematic review and meta-analysis. Nutrition Reviews, 79(12), 1321-1337. 10.1093/nutrit/nuaa146

Hall, K. D., Ayuketah, A., Brychta, R., Cai, H., Cassimatis, T., Chen, K. Y. \& Zhou, M. (2019). Ultra-Processed Diets Cause Excess Calorie Intake and Weight Gain: An Inpatient Randomized Controlled Trial of Ad Libitum Food Intake. Cell Metabolism, 30(1), 67-77. 10.1016/j.cmet.2019.05.008.

Harttig, U., Haubrock, J., Knüppel, S., Boeing, H.; \& EFCOVAL Consortium. (2011). The MSM program: web-based statistics package for estimating usual dietary intake using the Multiple Source Method. European Journal of Clinical Nutrition, 65 Suppl 1:S87-9. 10.1038/ejcn.2011.92

Instituto Brasileiro de Geografia e Estatística [IBGE]. (2011). Pesquisa de orçamentos familiares 2008-2009: análise do consumo alimentar pessoal no Brasil. Rio de Janeiro: IBGE. Disponível em: https://biblioteca.ibge.gov.br/visualizacao/livros/liv50063.pdf

Instituto Brasileiro de Geografia e Estatística [IBGE]. (2020). Pesquisa de orçamentos familiares 2017-2018: análise do consumo alimentar pessoal no Brasil. Rio de Janeiro: IBGE. Disponível em: https://biblioteca.ibge.gov.br/visualizacao/livros/liv101742.pdf

Jensen, T., Abdelmalek, M. F., Sullivan, S., Nadeau, K.J. Green, M. Roncal. C. \& Johnson, R. J. (2018). Fructose and sugar: A major mediator of non-alcoholic fatty liver disease. Journal of Hepatology, 68(5), 1063-1075. 10.1016/j.jhep.2018.01.019

Jiménez, L. G. \& Martín-moreno, M. (1995). Cuestionario de frecuencia de consumo alimentario. In: Majem LIS, Aranceta B, Verdú MJ. Nutrición y Salud Pública - Métodos, bases cientfflcas y aplicaciones. Espaiia: Masson.

Karnikowski, M., Córdova, C., Oliveira, R. J., Karnikowski, M. G., \& Nóbrega, O. T. (2007). Non-alcoholic fatty liver disease and metabolic syndrome in Brazilian middle aged and older adults. São Paulo Medical Journal, 125(6), 333-337. 10.1590/s1516-31802007000600006

Kirpich, I. A, Miller, M. E., Cave, M. C., Joshi-Barve, S., \& McClain, C. J. (2016). Alcoholic Liver Disease: Update on the Role of Dietary Fat. Biomolecules, 6(1), 1. 10.3390/biom6010001.

Lohman, T. G., Roche, A. F., \& Martorell, R. (1988). Anthropometric standardization reference manual. 1ed. Champaign: Human Kinetics Books. 
Lu, F. B., Hu, E. D., Xu, L. M., Chen, L., Wu, J. L., Li, H., Chen, D., \& Chen, Y. (2018). The relationship between obesity and the severity of non-alcoholic fatty liver disease: systematic review and meta-analysis. Expert Review Gastroenteroly Hepatoly, 12(5), 491-502. 10.1080/17474124.2018.1460202

McClave, S. A, Taylor, B. E, Martindale, R. G, Warren, M. M, Johnson, D. R, Braunschweig, C., \& Mckeever, L. (2016). American Society for Parenteral and Enteral Nutrition. Guidelines for the Provision and Assessment of Nutrition Support Therapy in the Adult Critically Ill Patient: Society of Critical Care Medicine (SCCM) and American Society for Parenteral and Enteral Nutrition (A.S.P.E.N.). Journal of Parenteral and Enteral Nutrition, 40(2), 159-211. 10.1002/jpen.2267

Mendez-Sanchez, N., Arrese, M., Gadano, A., Oliveira, C. P., Fassio, E., Arab, J. P. \& Silva, M. (2021). The Latin American Association for the Study of the Liver (ALEH) position statement on the redefinition of fatty liver disease. The Lancet Gastroenteroly \& Hepatology, 6(1):65-72. 10.1016/S2468-1253(20)30340$\mathrm{X}$

Mirizzi, A., Franco, I., Leone, C.M., Bonfiglio, C., Cozzolongo, R., Notarnicola, M. \& Osella, A. R. (2019). Effects of Some Food Components on NonAlcoholic Fatty Liver Disease Severity: Results from a Cross-Sectional Study. Nutrients, 11(11), 2744. 10.3390/nu11112744. PMID: 31726714.

Monteiro, A. C., Pfrimer, K., Tremeschin, M. H., Molina, M. C., Chiarello, P., \& Vannucchi, H. (2007). Consumo Alimentar: Visualizando Porções. 1ed. Rio de Janeiro: Guanabara Koogan.

Monteiro, C. A., Cannon, G., Levy, R., Moubarac, J. C., Jaime, P., Martins, A. P. \& Parra, D. (2016). NOVA. The star shines bright. Food classification. Public Health. World Nutrition Journal, 7(28-38). Disponível em: https://www.worldnutritionjournal.org/index.php/wn/article/view/5/4

Monteiro, C. A., Cannon, G., Levy, R. B., Moubarac, J. C., Louzada, M. L., Rauber, F. \& aime, P. C. (2019) Ultra-processed foods: What they are and how to identify them. Public Health Nutrition, 22(5), 936-941. 10.1017/S1368980018003762

Monteiro, C. A., Cannon, G., Moubarac, J. C., Levy, R. B., Louzada, M. L. C., \& Jaime, P. C. (2018). The UN Decade of Nutrition, the NOVA food classification and the trouble with ultra-processing. Public Health Nutrition, 21(1), 5-17. https://doi.org/10.1017/S1368980017000234

Moshfegh, A.J., Rhodes, D. G., Baer, D. J., Murayi, T., Clemens, J.C., Rumpler, W. V. \& Cleveland, L. E. (2008). The US Department of Agriculture Automated Multiple-Pass Method reduces bias in the collection of energy intakes. The American Journal of Clinical Nutrition, 88(2), 324-332. 10.1093/ajcn/88.2.324

Pagliai, G., Dinu, M., Madarena, M. P., Bonaccio, M., Iacoviello, L., \& Sofi, F. (2021). Consumption of ultra-processed foods and health status: a systematic review and meta-analysis. British Journal of Nutrition, 125(3), 308-318. https://doi.org/10.1017/S0007114520002688.

Pedraza, D. F. \& Menezes, T. N. (2015). Questionário de frequência alimentar desenvolvido e validado para a população brasileira: uma revisão da literatura. Ciencia e Saúde Coletiva, 20(9), 2697-2720. 10.1590/1413-81232015209.12602014

Pinheiro, A. V. B., Lacerda, E. M. A., Benzecry, E. H., Gomes, M. C. S., \& Costa, V. M. (2005). Tabela para avaliação do consumo alimentar em medidas caseiras. 5ed. São Paulo: Atheneu.

Poti, J.M., Braga, B., \& Qin, B. (2017). Ultra-processed Food Intake and Obesity: What Really Matters for Health-Processing or Nutrient Content? Current Obesity Reports, 6(4), 420-431. 10.1007/s13679-017-0285-4

Rico-Campa, A., Martinez-Gonzalez, M. A., Alvarez-Alvarez, I., de Deus, M. R., de la Fuente-Arrillaga, C., Gómez-Donoso, C., \& Bes-Rastrollo, M. (2019). Association between consumption of ultra-processed foods and all cause mortality: SUN prospective cohort study. BMJ, 365, 11949. 10.1136/bmj.11949.

Sanyal, A. J., Brunt, E. M., Kleiner, D. E., Kowdley, K. V., Chalasani, N., Lavine, J. E., Ratziu, V., \& McCullough, A. (2011). Endpoints and clinical trial design for nonalcoholic steatohepatitis. Hepatology, 54(1), 344-353. 10.1002/hep.24376

Sarmento, R. A., Riboldi, B. P., da Costa Rodrigues, T., de Azevedo, M. J., \& de Almeida, J. C. (2013). Development of a quantitative food frequency questionnaire for Brazilian patients with type 2 diabetes. BMC Public Health, 13(740). 10.1186/1471-2458-13-740.

Shiha, G., Korenjak, M., Eskridge, W., Casanovas, T., Velez-Moller, P., Högström, S. \& Eslam, M. (2021). Redefining fatty liver disease: an international patient perspective. The Lancet Gastroenteroly \& Hepatology, 6(1), 73-79. 10.1016/S2468-1253(20)30294-6

Slater, B., Philippi, S. T., Marchioni, D. M. L., \& Fisberg, R. M. (2003). Validação de Questionários de Frequência Alimentar-QFA: considerações metodológicas. Revista Brasileira de Epidemiologia, 6(3): 200-208. Disponível em: https://www.scielosp.org/article/rbepid/2003.v6n3/200-208/

Sofi, F., Giangrandi, I., Cesari, F., Corsani, I, Abbate, R., Gensini, G. F., \& Casini, A. (2010). Effects of a 1-year dietary intervention with n-3 polyunsaturated fatty acid-enriched olive oil on non-alcoholic fatty liver disease patients: a preliminary study. International Journal of Food Sciences, 61(8), 792-802. $10.3109 / 09637486.2010 .487480$

Tutunchi, H., Saghafi-Asl, M., Asghari-Jafarabadi, M., \& Ostadrahimi, A. (2021). Association between Dietary Patterns and Non-alcoholic Fatty Liver Disease: Results from a Case-Control Study. The Archives of Iranian Medicine, 24(1), 35-42. 10.34172/aim.2021.06

Verly Junior, E., Cesar, C. L., Fisberg, R. M., \& Marchioni, D. M. (2013). Variância intrapessoal da ingestão de energia e nutrientes em adolescentes: correção de dados em estudos epidemiológicos. Revista Brasileira de Epidemiologia, 16(1), 170-177. https://doi.org/10.1590/S1415-790X2013000100016

Wang, C., Horby, P. W., Hayden, F. G., \& Gao, G. F. A novel coronavirus outbreak of global health concern. (2020). Lancet, 395(10223), 470-473. $10.1016 /$ S0140-6736(20)30185-9

Willett, W. C. \& Stampfer, M. J. (1986). Total energy intake: implieations for epidemiologieal analyses. American Journal of Epidemiology, 124(1), 17-27. 10.1093/oxfordjournals.aje.a114366.

World Health Organization [WHO] [Internet]. Emergency situation reports. Geneva: WHO. https://www.who.int/emergencies/situation-reports.

World Health Organization [WHO]. (1998). Obesity: preventing and managing the global epidemic. Report of a World Health Organization Consultation. 1ed. Geneva: World Health Organization. 
Research, Society and Development, v. 10, n. 16, e280101623506, 2021

(CC BY 4.0) | ISSN 2525-3409 | DOI: http://dx.doi.org/10.33448/rsd-v10i16.23506

Younossi, Z.M., Tacke, F., Arrese, M., Chander, S. B., Mostafa I, Bugianesi E. \& Vos, M. B. (2019). Global Perspectives on Nonalcoholic Fatty Liver Disease and Nonalcoholic Steatohepatitis. Hepatology, 69(6), 2672-2682. 10.1002/hep.30251

Younossi, Z. M., Koenig, A. B., Abdelatif, D., Fazel, Y., Henry, L., \& Wymer, M. (2016). Global epidemiology of nonalcoholic fatty liver disease-meta-analytic assessment of prevalence, incidence, and outcomes. Hepatology, 64(1), 73-84. 10.1002/hep.28431 